\title{
Pulsational line profile variation of the roAp star HR $3831^{\star}$
}

\author{
O. Kochukhov
}

Department of Astronomy and Space Physics, Uppsala University, 75120 Uppsala, Sweden

e-mail: oleg@astro.uu.se

Received 30 April 2005 / Accepted 12 September 2005

\section{ABSTRACT}

We report the first comprehensive investigation of the line profile variation caused by non-radial pulsation in an oscillating magnetic chemically peculiar star. Spectrum variation of the well-known roAp star HR 3831 was detected using very high-resolution high signal-to-noise spectroscopic time-series observations and was followed through the whole rotation cycle of the star. We confirmed the outstanding diversity of pulsational behaviour of different lines in the HR 3831 spectrum and attributed this phenomenon to an interplay between extreme vertical chemical inhomogeneity of the HR 3831 atmosphere and a running pulsation wave, that is propagating towards the upper photospheric layers with increasing amplitude. The rapid profile variation of the Nd III $6145 \AA$ line, which shows the maximum pulsational disturbances in the studied wavelength region, is characterised by measuring changes of its equivalent width and the first three moments. Each of these observables exhibits almost purely sinusoidal variation through the pulsation cycle, with the amplitude and phase clearly modulated by the stellar rotation. In contrast to previous photometric pulsational measurements of roAp stars, our spectroscopic observational material admits straightforward astrophysical interpretation and, hence, opens new and exciting possibilities for direct and accurate analysis of the roAp pulsations. We demonstrate that rotational modulation of the radial velocity oscillations cannot be fully explained by an oblique axisymmetric dipole $(\ell=1, m=0)$ mode, implied by the classical oblique pulsator model of roAp stars. Pulsational variation of the higher moments, in particular the line width, reveals substantial contribution of the high-order $(\ell=3)$ spherical harmonics that appear due to distortion of pulsations in HR 3831 by the global magnetic field. We interpreted observations with a novel numerical model of the pulsational variation and rotational modulation of the line profile moments in roAp stars. Comparison of the observed and computed radial velocity and line width variation was used to establish parameters of the oblique pulsator model of HR 3831. Furthermore, definite detection of pulsational variation in lines of light and iron-peak elements allows the first 3-D mapping of pulsations in a non-radially oscillating star.

Key words. line: profiles - stars: chemically peculiar - stars: oscillations - stars: individual: HR 3831

\section{Introduction}

\subsection{RoAp stars}

The rapidly oscillating Ap (roAp) stars are cool, magnetic, chemically peculiar stars, that pulsate in high-overtone nonradial acoustic $p$-modes. There are 34 roAp stars known at the present time (Kurtz \& Martinez 2000 for a recent review). These objects oscillate with periods in the range of 6-21 min (Kurtz \& Martinez 2000; Elkin et al. 2005), while their light variation amplitudes rarely exceed $10 \mathrm{mmag}$ in Johnson $B$. Pulsations in roAp stars bear a certain resemblance to the solar acoustic $p$-mode oscillations, but are characterised by a smaller number of excited modes and have amplitudes that are three orders of magnitude larger than the $p$-mode oscillations in the Sun. Photometric investigations of roAp stars were carried out during the last 25 years and have yielded unique asteroseismic information on the internal structure and fundamental

* Based on observations obtained at the European Southern Observatory, La Silla, Chile (ESO program No. 66.D-0241A). parameters of roAp pulsators (e.g., Matthews et al. 1999; Cunha et al. 2003).

Since the discovery of roAp pulsations by Kurtz (1978), it has become clear that strong magnetic fields in these stars play a defining role in exciting the oscillations and shaping the main pulsation properties. It was found that the amplitude and phase of the rapid light variation are modulated with the stellar rotation and that the phases of the magnetic field and pulsation amplitude extrema typically coincide with each other (Kurtz 1982). Detailed frequency analyses of the roAp photometric light curves convincingly demonstrated that the main characteristics of their rapid brightness variation can be attributed to the oblique axisymmetric dipole $(\ell=1, m=0)$ modes, aligned with the axis of a nearly axisymmetric quasidipolar magnetic field. This oblique pulsator model, first proposed by Kurtz (1982), gave rather successful and straightforward phenomenological explanation of the main features in the roAp frequency spectra. However, subsequent studies of roAp pulsations have revealed that the mode geometry in some stars defies a simple interpretation in terms of a single spherical harmonic (e.g., Kurtz et al. 1989). Moreover, 
gradual accumulation of the photometric information about pulsations in some of the best-studied monoperiodic roAp stars with oblique dipole modes has provided evidence of significant deviations in the mode geometry from the expected axisymmetric dipole shape - an effect loosely called "distorted dipole modes" (Kurtz et al. 1997).

Several theoretical investigations (e.g., Dziembowski \& Goode 1985; Shibahashi \& Takata 1993; Cunha \& Gough 2000) have argued that the combined effects of the magnetic field and rotation may be responsible for the surface geometry of pulsational perturbations departing from a pure spherical harmonic form. In fact, recent theoretical study by Bigot \& Dziembowski (2002) challenges the general framework of the classical oblique pulsator model of Kurtz (1982) by showing that accurate non-perturbative treatment of the interaction between stellar rotation, pulsations, and magnetic field may lead to an entirely new geometrical picture of the roAp oscillations. Calculations by Bigot \& Dziembowski (2002) suggest that pulsation eigenmodes are represented by a complex superposition of spherical harmonic functions and may contain substantial non-axisymmetric components. Furthermore, strong effects due to centrifugal force are expected to break the locking of the pulsation axis with the dipolar magnetic field, so that pulsations are aligned with neither the magnetic nor the rotation axes. It should be noted, however, that these calculations are limited to the $p$-mode pulsations in stars with magnetic field strength $\lesssim 1 \mathrm{kG}$ and are not directly applicable to the majority of roAp pulsators, which have surface magnetic fields of a few $\mathrm{kG}$.

In contrast to the approach followed by Bigot \& Dziembowski (2002), Saio \& Gautschy (2004) and Saio (2005) study the effects of strong magnetic field on axisymmetric $p$-mode oscillations assuming an alignment between pulsations and dipolar magnetic field and disregarding distortion of pulsation modes by the stellar rotation. Saio \& Gautschy (2004) find substantial deviation of the surface structure of pulsational fluctuations from a single spherical harmonic. In particular, the $\ell=1$ mode is distorted by a dipolar magnetic field in such a way that pulsations are described by superposition of the spherical harmonics with odd angular degree $\ell$, and pulsation amplitude is strongly confined to the magnetic axis in the outer stellar layers.

In view of the diversity and increasing sophistication of the theoretical speculations about the geometry and physics of roAp oscillations, it becomes apparent that modelling of the photometric light curves of roAp stars is generally insufficient for accurately determining the structure of pulsation modes and is unable to provide useful tests of theoretical predictions. The information content of the high-speed photometric observations is small due to averaging pulsational disturbances over the visible stellar hemisphere (see Saio \& Gautschy 2004) and highly uncertain because rapid light variation in roAp stars involves non-linear and non-adiabatic effects that are not at all understood (Watson 1988; Medupe 2002; Saio 2005). It has been argued (e.g., Kurtz 1990) that spectroscopic timeresolved observations, in particular investigations of pulsational variation in the Doppler-broadened spectral line profiles at different rotation phases, can potentially provide much more precise and unprecedentedly complete information about the vertical and horizontal structure of $p$-modes and about its relation to the magnetic field topology, chemical inhomogeneities, and anomalous atmospheric structure of Ap stars. Such spectroscopic studies will eventually allow us to address the underlying questions about the physics of pulsating peculiar stars, for instance, the problem of excitation of the magnetoacoustic oscillations or the nature of the mode selection mechanism.

\subsection{Spectroscopic studies of the roAp pulsations}

Until recently our knowledge of the $p$-modes oscillations in roAp stars was essentially based on high-speed photometric observations. Early time-resolved spectroscopic studies (Matthews et al. 1988; Libbrecht 1988) were focused on detecting pulsational radial velocity (RV) changes and deriving the ratio of RV and photometric amplitude. Subsequent low spectral resolution investigations of $\alpha$ Cir and HR 3831 by Baldry et al. $(1998 \mathrm{a}, \mathrm{b})$ revealed unexpected diversity of the pulsational amplitude and phase in different wavelength regions, showing that for roAp stars the concept of a "typical" amplitude of RV oscillations is meaningless. Baldry et al. (1999) and Baldry \& Bedding (2000) also found a systematic change in pulsational amplitude and phase in the wings of the hydrogen $\mathrm{H} \alpha$ line, and suggested that this behaviour originates in a strong depth-dependence of the pulsational characteristics in roAp atmospheres.

High-resolution observations of $\gamma$ Equ by Kanaan \& Hatzes (1998) localised RV variations of individual metal lines and confirmed that pulsations change dramatically from one spectral line to another. This phenomenon remained a mystery, however, because no clear underlying systematic trends or dependencies were identified. A breakthrough discovery was made by Savanov et al. (1999), who demonstrated that the largest pulsational RV shifts of up to $1 \mathrm{~km} \mathrm{~s}^{-1}$ in $\gamma$ Equ occur in the lines of Pr III and Nd III. Very high spectral resolution timeresolved follow-up observations of this star by Kochukhov \& Ryabchikova (2001a) confirmed these results and also revealed the phase shifts between the variations of singly and doubly ionized lines of the rare-earth elements (REE). Our study emphasised a stunning discrepancy (sometimes up to two orders of magnitude in RV amplitude!) between strong pulsations in REE lines and the absence of changes in the lines of light and iron-peak elements, which do not show oscillations above a few tens of $\mathrm{m} \mathrm{s}^{-1}$. Discovery of the pulsational spectroscopic variability in $10 \mathrm{Aql}$ (Kochukhov et al. 2002) and the first analyses of the variation in individual metal lines in $\alpha$ Cir and HR 3831 (Kochukhov \& Ryabchikova 2001b) showed that this unusual pulsational behaviour, dominated by the REE lines, is not limited to $\gamma \mathrm{Equ}$, but is present in a very similar form in other roAp stars. This finding was further strengthened by the wide wavelength region analyses of HR 1217 (Balona \& Zima 2002), HR 3831 (Balona 2002), $\alpha$ Cir (Balona \& Laney 2003), 33 Lib (Mkrtichian et al. 2003), and HD 166473 (Kurtz et al. 2003).

A plausible explanation was suggested by Ryabchikova et al. (2002) for the nature of pulsational spectroscopic variation in roAp stars. This study of the atmospheric structure of 
$\gamma$ Equ found compelling evidence of the strong vertical separation (stratification) of chemical elements. Under the influence of radiative diffusion, light and iron-peak elements are accumulated at the bottom of the $\gamma$ Equ atmosphere, whereas REEs are pushed high above the normal line-forming region. Ryabchikova et al. (2002) proposed that the atmospheric layers contributing to the absorption in REE lines are located substantially higher than the formation depths of other elements. The most recent detailed non-LTE analysis by Mashonkina et al. (2005) and Ryabchikova (2005) indicates that the Nd II and Nd III lines in $\gamma$ Equ originate from the optical depths $10^{-4}-10^{-5}$. These high atmospheric layers, which are rich in REE, are characterised by enhanced amplitude of non-radial pulsations. Within the framework of this model, the phase shifts between variations in different lines are attributed to a running magnetoacoustic wave propagating outwards in the stellar atmosphere and increasing in amplitude with height. Similarities between different roAp stars in spectroscopic pulsational behaviour suggest that they share similar vertical chemical and pulsational profiles. The method of tomographic mapping of the vertical structure of roAp pulsation modes, pioneered by Ryabchikova et al. (2002) and Kochukhov (2003), uses vertical chemical inhomogeneities as spatial depth filters and aims at reconstructing detailed vertical cross-sections of pulsational perturbations in roAp atmospheres, as can be done for no other type of main sequence, pulsating star.

Despite dramatic recent progress in understanding the vertical structure of the roAp pulsation modes and the interplay between pulsations and vertical stratification of chemical elements, relatively little attention has been paid to the problem of inferring the horizontal geometry of pulsations in roAp stars. The question of systematic mode identification, central to the studies of other types of pulsating stars, has not been thoroughly investigated in the case of magnetic pulsators. To a large extent, such an unsatisfactory situation is explained by the absence of suitable observational material. Studies of the vertical structure of a pulsating cavity capitalise on the extreme vertical inhomogeneity of the roAp atmospheres and typically make use of a relatively low $S / N$ spectroscopic time series, just sufficient for detecting pulsations and merely deducing amplitudes and phases of the RV changes in individual spectral lines. In contrast, probing the horizontal structure of pulsation modes is necessarily based on observations of the pulsational line profile variation (LPV) and, therefore, requires spectroscopic data of outstanding quality. Such observations so far have been presented only for one roAp star, $\gamma$ Equ (Kochukhov \& Ryabchikova 2001a). We investigated variability of the Pr III $6160 \AA$ and Nd III $6145 \AA$ lines for this star and probed the horizontal structure of the magnetoacoustic $p$-mode oscillation using several mode identification methods. Most important, it was convincingly shown that the moment variation of the REE lines is inconsistent with the picture that is expected for axisymmetric $(m=0)$ modes of low degree. This discovery of non-axisymmetric pulsations in a roAp star may give credibility to the generalised oblique pulsator model of Bigot \& Dziembowski (2002) and demonstrates the enormous diagnostic potential of the high-precision time-resolved spectroscopy of roAp stars.
Despite its interesting pulsational properties, $\gamma$ Equ is not an ideal target for mapping the horizontal mode structure because its extremely slow rotation does not allow useful constraints to be put on the surface chemical inhomogeneities and to explore oscillations from different geometrical aspects. Additional complications are introduced by multiperiodicity and short life time of the $p$-modes in $\gamma$ Equ (Martinez et al. 1996). Evidently, a more fruitful investigation of the LPV due to non-radial oscillation can be carried out for a monoperiodic roAp star with a high amplitude of pulsational spectral variation and a reasonably short rotation period. Both requirements are fulfilled for the well-known roAp star HR 3831. The present paper concentrates on this object and presents the first comprehensive study of the pulsational LPV in a roAp star.

\section{3. $H R 3831$}

HR 3831 (HD 83368, IM Vel) is one of the best-studied rapidly oscillating Ap pulsators. This star is a brighter component in a visual binary with a separation of 3.29" and a $\Delta V=2.84$ mag difference in brightness. HR 3831 has a visual magnitude of $V=6.25$ (Hurly \& Warner 1983) and belongs to the $\mathrm{SrCrEu}$ group of Ap stars (Houck 1978). The mean longitudinal magnetic field of HR 3831 was measured by Thompson (1983) and Mathys (1991). The longitudinal field varies sinusoidally with an amplitude of $737 \mathrm{G}$ and a mean value close to zero. The rotation period of $P_{\text {rot }}=2.851962 \mathrm{~d}$ was derived from the magnetic measurements.

Spectroscopic studies of HR 3831 were conducted by Polosukhina et al. (2000) and Shavrina et al. (2001). They analysed abundances of several chemical elements and studied strong rotational modulation of the profile of the Li I resonance doublet.

Kochukhov et al. $(2003,2004)$ carried out the first abundance Doppler Imaging of HR 3831 using spectra analysed in the present study and additional high-resolution observations. We combined Hipparcos parallax, Strömgren, and $\mathrm{H} \beta$ photometric indices, along with results of the model atmosphere analysis of the hydrogen lines in HR 3831 to derive fundamental stellar parameters: $T_{\text {eff }}=7650 \mathrm{~K}, \log g=4.2$, $\log L / L_{\odot}=1.091, R / R_{\odot}=2.003$, and inclination angle of the stellar rotation axis $i=68.2^{\circ}$. It was found that many lines in the HR 3831 spectrum show noticeable variation with rotation phase, implying that strong horizontal abundance gradients are present at the stellar surface. In particular, the Doppler inversions showed $\mathrm{Li}$ and Eu to be concentrated in pairs of opposite small spots, located at the stellar rotational equator, whereas $\mathrm{O}$ exhibits an extended overabundance area along the big circle perpendicular to the rotational equator. In the context of the present study, the behaviour of the doubly ionized $\mathrm{Nd}$ and Pr lines is of special interest because these REE lines turn out to be ideal pulsation indicators and are extensively analysed in the time-resolved spectra of roAp stars. Kochukhov et al. (2004) demonstrated that Nd III and Pr III lines do not show strong profile anomalies except for the rotation phases close to magnetic crossover $(\varphi=0.25,0.75)$, when pronounced doubling is evident in both the $\mathrm{Nd}$ and Pr absorption features (see also 
Kochukhov \& Ryabchikova 2001b). Our Doppler mapping of HR 3831 attributed this phenomenon to a ring of relative REE underabundance - a surface distribution that is the opposite of oxygen.

The horizontal abundance structures observed in HR 3831 are understood to be a result of the radiatively driven diffusive separation of chemical species in the presence of a dipolar magnetic field inclined at a large angle $\beta$ with respect to the rotation axis of the star. This magnetic topology is in perfect agreement with the parameters of the dipolar model $\left(\beta=86.8^{\circ}\right.$, $B_{\mathrm{p}}=2.49 \mathrm{kG}$ ) determined by Kochukhov et al. (2004), but contradicts the very large $11 \mathrm{kG}$ mean quadratic field measured in HR 3831 by Mathys (1995). However, the latter measurements may be severely biased by neglect of surface chemical inhomogeneities in the quadratic field diagnostic procedure (see Sect. 4 in Kochukhov et al. 2004).

Non-radial pulsations in HR 3831 were discovered and investigated by Kurtz (1982). Subsequent analysis of the pulsational behaviour, using photometric data spanning four observing seasons, was presented by Kurtz et al. (1997). They revealed strong rotational modulation of pulsations and determined a principal pulsation frequency $v=1428.0091 \mu \mathrm{Hz}$ (11.67 $\mathrm{min})$. The power spectrum around the main frequency is described by a septuplet structure with a separation of the frequency components by $v_{\text {rot }}=4058.265 \mathrm{nHz}$, corresponding to the stellar rotational period $P_{\text {rot }}=2.851976 \pm$ $0.00003 \mathrm{~d}$. Pulsational light variation of HR 3831 is clearly non-sinusoidal, with the first-harmonic quintuplet and the second-harmonic triplet unambiguously detectable in the Fourier transform of the time-resolved photometric data.

A preliminary discovery report of the pulsational variations in the individual metal spectral lines in HR 3831 was published by Kochukhov \& Ryabchikova (2001b). In this study we demonstrated that, similar to the pulsations in other roAp stars, the doubly ionized REE lines in HR 3831 are distinguished by high-amplitude RV variation during the pulsation cycle of the star. This was confirmed by Balona (2002), who made highresolution time-series observations in a wide spectral region at several rotation phases of HR 3831. Balona found that all metal lines with large pulsational variability are invariably associated with the Pr III and Nd III spectral features. He also detected a strong decrease in the amplitude of the pulsational RV variation from $\mathrm{H} \alpha$ to the higher members of the Balmer series, which supports the idea that the amplitude of non-radial oscillation rapidly changes with height in the atmospheres of roAp stars.

The quality of the observational material collected by Balona (2002) was not sufficient for detecting and studying pulsational changes in the profiles of individual spectral lines. Instead, Balona attempted to use a cross-correlation function derived from the Nd III lines for tentative mode identification. He suggested that the two frequencies detected in the RV variability can be attributed to the $\ell=2, m=0$ and $\ell=2, m=-2$ non-radial modes aligned with the stellar rotation axis. This mode identification remains controversial since it was not supported by any quantitative analysis (e.g., line profile calculations) and clearly contradicts the well-known dipole oblique pulsator model required to explain the rapid photometric variability of HR 3831 .
Table 1. Observing log of the time-resolved spectroscopic monitoring of HR 3831. Columns give the UT date, heliocentric Julian Date for the beginning and end of observation, the duration of continuous monitoring in hours, the range of rotation phases calculated according to the ephemeris of Kurtz et al. (1997), and the number of spectra obtained each night.

\begin{tabular}{ccccc}
\hline \hline Date & HJD-2451900 & $\Delta T$ & $\varphi$ & $N$ \\
\hline $05 / 02 / 2001$ & $45.5533-45.8779$ & 7.79 & $0.965-0.079$ & 300 \\
$06 / 02 / 2001$ & $46.5568-46.8796$ & 7.75 & $0.317-0.430$ & 300 \\
$07 / 02 / 2001$ & $47.5470-47.8678$ & 7.70 & $0.664-0.776$ & 300 \\
$13 / 02 / 2001$ & $53.5328-53.8786$ & 8.30 & $0.763-0.884$ & 320 \\
$14 / 02 / 2001$ & $54.5286-54.8716$ & 8.23 & $0.112-0.232$ & 320 \\
$15 / 02 / 2001$ & $55.5209-55.8678$ & 8.33 & $0.460-0.581$ & 320 \\
\hline
\end{tabular}

A new pulsation Doppler Imaging method of resolving the horizontal structure of stellar non-radial oscillations was developed by Kochukhov (2004a). This technique uses highresolution observations of LPV and permits direct mapping of the surface pulsation velocity amplitude without an a priori parameterisation of pulsation with spherical harmonic functions. Preliminary results of the application of this promising method to HR 3831 were reported by Kochukhov (2004b). Doppler mapping of pulsations in HR 3831 was based on the spectra analysed here and suggested that the distorted dipole oscillation mode is inclined at a large angle with respect to the stellar rotation axis and is aligned with the dipole component of the stellar magnetic field.

\section{Observations and spectra reduction}

Very high-resolution spectroscopic time-series observations of HR 3831 presented in this paper were collected in February 2001 with the Very Long Camera of the Coudé Echelle Spectrograph (CES) fibre-linked to the Cassegrain focus of the ESO 3.6-m telescope. The spectrograph was configured to reach a resolving power of $\lambda / \Delta \lambda=123500$ with the help of the medium resolution CES image slicer. The detector, the ESO CCD-61 $2 \mathrm{~K} \times 4 \mathrm{~K}$ chip, allowed us to record a $40.4 \AA$ spectral interval centred at $\lambda 6136 \AA$.

During the CES observations, we continuously monitored HR 3831 for about $8 \mathrm{~h}$ on each of the 6 observing nights with a break of 5 nights in the middle of the run. Such an observing strategy enabled us to obtain 300-320 stellar spectra each night and ensure a good coverage of the rotation period of HR 3831, with phase gaps not exceeding $8 \%$ of the rotation cycle (see a log of observations in Table 1). A total of 1860 spectra was collected for HR 3831. Observations were obtained with a $70^{\mathrm{s}}$ exposure time, whereas approximately $20^{\mathrm{s}}$ was spent on reading the CCD detector and file transfer. The dead time was minimised by exposing only half of the detector in the cross-dispersion direction and additionally binning by a factor of four in the same direction. This setup allowed us to observe HR 3831 with a $78 \%$ duty cycle and proved to be an appropriate compromise between a reasonable sampling of the pulsation period and reaching a sufficient signal-to-noise ratio in individual spectra. Then, $60^{\mathrm{s}}$ exposures of the ThAr comparison spectrum were obtained every $1.3-1.4 \mathrm{~h}$ during observations. 


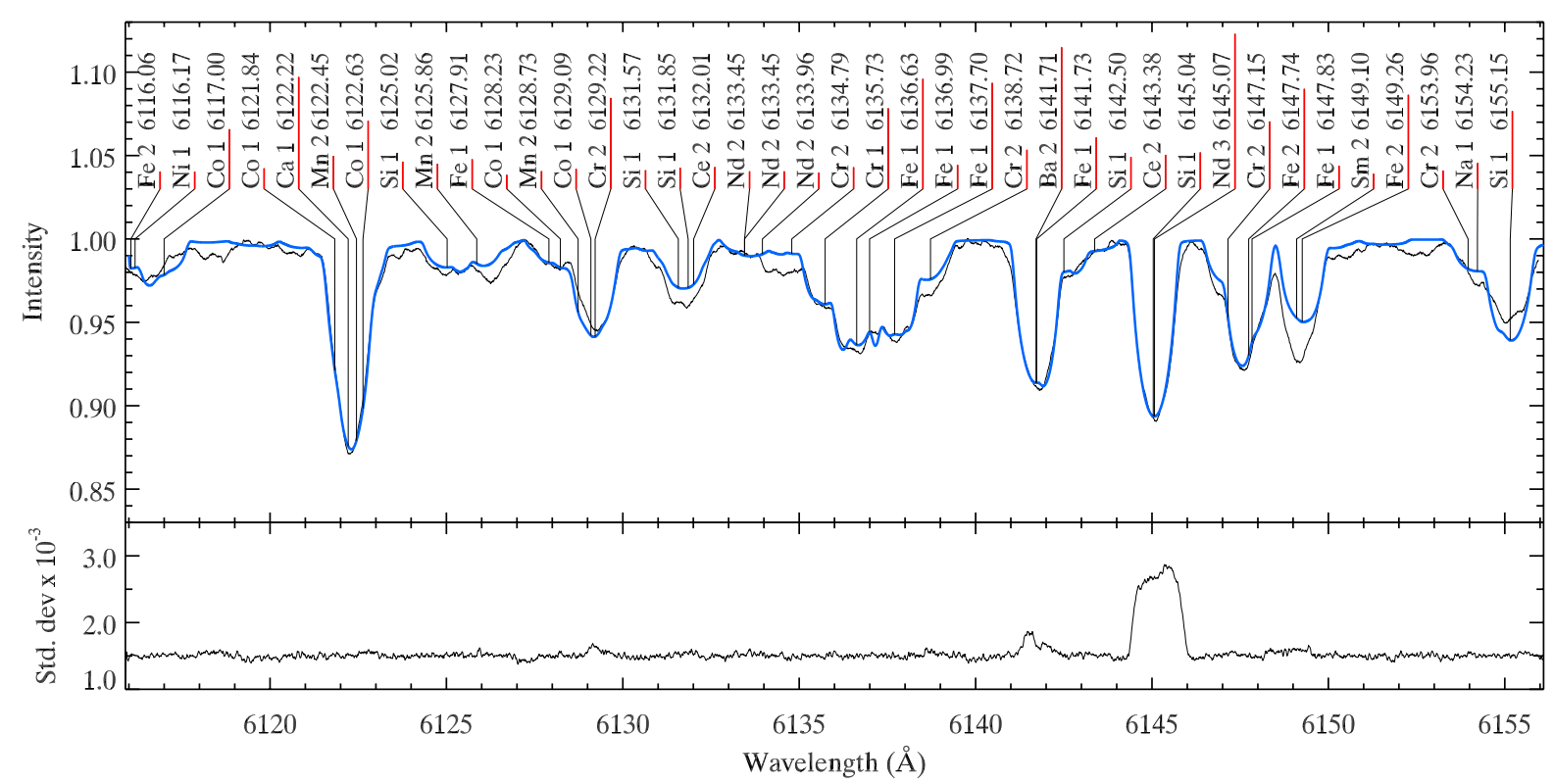

Fig. 1. The upper panel compares the mean spectrum of HR 3831 (thin line) and magnetic spectrum synthesis calculations (thick line) in the 6116-6156 A wavelength region. The strongest spectral lines are identified. The length of the lines under the identification is proportional to the strength of the corresponding spectral features. The lower panel shows the standard deviation spectrum.

A dedicated set of IDL computer routines was developed to perform optimal reduction of the CES spectra based on the strategy and procedures implemented in the REDUCE package by Piskunov \& Valenti (2002). The reduction steps included construction of the master flat field and bias frames, subtraction of bias and correction of the target spectra by the normalised flat field, subtraction of background, extraction of 1-D spectra, and correction for the overall curvature of the spectra in the dispersion direction. Since the CES instrument was designed to record observations at a resolving power of up to $\lambda / \Delta \lambda=235000$, its instrumental profile is oversampled in the medium resolution mode $(\approx 5$ pixels per resolution element). We took advantage of this situation and resampled all spectra in the dispersion direction, thereby decreasing the total number of pixels by a factor of two. This procedure yielded 1-D spectra with $S / N$ in the range of $110-150$ per pixel.

A special procedure was developed to establish the wavelength scale and to monitor possible instrumental drifts of the spectrograph during observing nights. We identified and measured positions of 23 emission lines in all the available ThAr spectra and then fitted the pixel-wavelength relation with a third-order polynomial. Comparison spectra obtained on the same nights were analysed simultaneously to derive both common higher order polynomial coefficients and a time-dependent instrumental shift (the lowest order polynomial coefficient). The final internal accuracy of the wavelength scale established through this procedure was $\approx 8 \times 10^{-5}\left(4 \mathrm{~m} \mathrm{~s}^{-1}\right)$. We found that the zero point of the CES spectrograph exhibited an overall smooth drift of $60-180 \mathrm{~m} \mathrm{~s}^{-1}$ due to changes in ambient temperature and pressure during observations of HR 3831. At the same time, the average shift between the consecutive ThAr spectra taken 1.3-1.4 hours apart was at the level of $17-35 \mathrm{~m} \mathrm{~s}^{-1}$. This confirms an excellent short-term stability of the CES instrument and suggests that possible random instrumental shifts do not exceed $\sim 10 \mathrm{~m} \mathrm{~s}^{-1}$. Consequently, it was justified to linearly interpolate the zero point between the comparison spectra taken before and after groups of stellar exposures. Finally, the spectra were shifted to the heliocentric reference frame.

In the last reduction stage, the individual spectra of HR 3831 were normalised to the continuum. This procedure was carried out in a series of steps in order to ensure consistent normalisation of all stellar spectra. First, a third order polynomial was fitted through the continuum points of the nightly mean spectra. This average continuum profile was applied to individual observations and was subsequently corrected for each spectrum by the heavily smoothed ratio of the average and individual spectra. A similar procedure was followed to achieve homogeneity of the continuum normalisation of the spectra obtained on different nights.

\section{Spectrum synthesis and line identification}

The line identification in the 6116-6156 ̊ wavelength region of HR 3831 was carried out based on magnetic spectrum synthesis calculations. We adopted the model atmosphere parameters $T_{\text {eff }}=7650 \mathrm{~K}, \log g=4.2$, and the projected rotational velocity $v_{\mathrm{e}} \sin i=33 \mathrm{~km} \mathrm{~s}^{-1}$ determined by Kochukhov et al. (2004). The relevant parameters of atomic transitions were extracted from the VALD database (Kupka et al. 1999) and were fine-tuned further by fitting the solar atlas. Synthetic spectra were calculated using the polarised radiative transfer code SYNTHMAG (Piskunov 1999). A homogeneous magnetic distribution was adopted, and a field strength of $B_{\mathrm{s}}=1.75 \mathrm{kG}$ was estimated from the dipolar magnetic field model derived for HR 3831 by Kochukhov et al. (2004).

The comparison between the average spectrum of HR 3831 and our theoretical calculation is illustrated in Fig. 1. In general, we find a satisfactory agreement. Remaining discrepancies are attributed to averaging over spotted surface 



Fig. 2. Illustration of the rapid variation of the Nd III $6145.07 \AA$ line in the spectrum of HR 3831. The upper panels show LPV recorded on the night of 5 Feb. 2001 and the lower panels those obtained on 7 Feb. 2001. Each of the six horizontal subpanels presents the average spectrum of HR 3831 at the specified rotation phase and time sequence of the difference between the average and 50 individual time-resolved observations obtained during 74 min and covering roughly 6 pulsation periods of HR 3831. Consecutive difference spectra are shifted in the vertical direction. The bottom part of the horizontal subpanels shows the standard deviation for each pixel of the observed spectra. abundance structures (not accounted for in our spectrum synthesis) and unidentified spectral lines missing in the theoretical spectrum.

In the previous time-resolved observations of the $\lambda 6150 \AA$ region in slowly rotating roAp stars (Kochukhov \& Ryabchikova 2001a; Kochukhov et al. 2002), we were able to study several dozen spectral lines. Unfortunately, a moderately rapid rotation of HR 3831 makes a detailed pulsation analysis possible only for a few relatively unblended spectral features and for blends with well-defined components. Those included the CaI 6122.22, Cr II 6129.22 $\AA$ lines, the blend of $\mathrm{SiI}$ and $\mathrm{Ce}$ II at $\lambda 6131.9 \AA$, the Ba II 6141.71, Nd III 6145.07, Fe II 6147.74, and 6149.26 $\AA$ lines. Note that the last Fe II line is severely blended with an unidentified REE line at $\lambda 6148.85 \AA$, which shows a high-amplitude pulsation variability in the spectrum of $\gamma$ Equ (Kochukhov \& Ryabchikova 2001a) and other sharp line roAp stars.

\section{Pulsational behaviour of the $\mathrm{Nd}$ III $6145.07 \AA$ line}

\subsection{Observations of the rapid line profile variability}

The high quality of the spectroscopic observational data obtained for HR 3831 enables a direct detection and comprehensive analysis of the LPV in REE spectral lines. The strong line of doubly ionized neodymium at $\lambda 6145.07 \AA$ was found to exhibit rapid line profile changes that are clearly modulated with the stellar rotation. Figure 2 illustrates variability of this $\mathrm{Nd}$ III line at two representative rotation phases. At the phase of magnetic extremum, $\varphi=0.02$ (the upper panels in Fig. 2), the average profile of the $\mathrm{Nd}$ feature is not significantly distorted by the inhomogeneous surface distribution of this element. Pulsational variability has a simple symmetric pattern, as expected for the oblique dipole pulsation mode. The line profile as a whole becomes skewed to the red and then to the blue. This is very different from the pulsational spectroscopic behaviour of some of the hot non-radial pulsators, which is characterised by high-contrast bumps and dips moving across stellar line profiles (e.g. Schrijvers et al. 2004). Such behaviour is not observed in HR 3831 because of the low angular degree $\ell$ of the excited mode. The dominant pulsational contribution comes from the global $\ell=1-3$ mode, which distorts a significant part of the line profile, not unlike the spectral changes typical of the radial stellar pulsation (e.g. Telting et al. 1997).

In contrast to the phase of magnetic extremum, pulsational changes of the Nd III line at the crossover phase (lower panels in Fig. 2) have lower amplitude and are more complex. According to the surface Doppler maps of HR 3831 published by Kochukhov et al. (2004), rotation phase $\varphi=0.72$ 

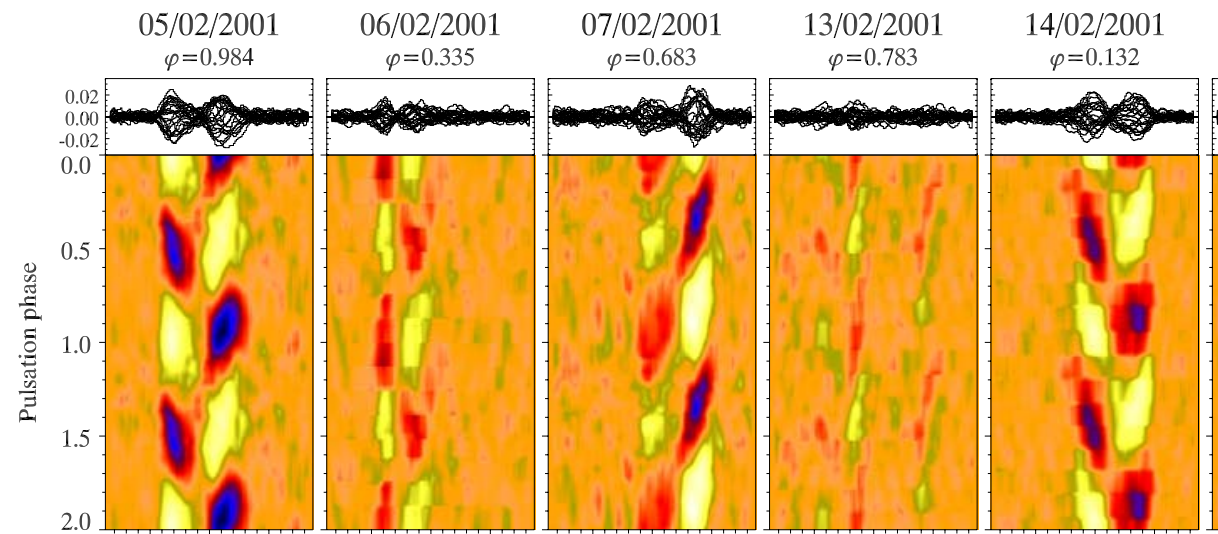

$15 / 02 / 2001$

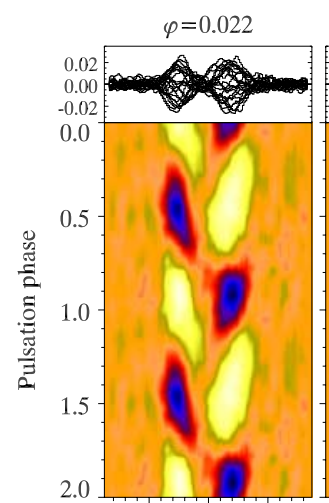

$\varphi=0.374$

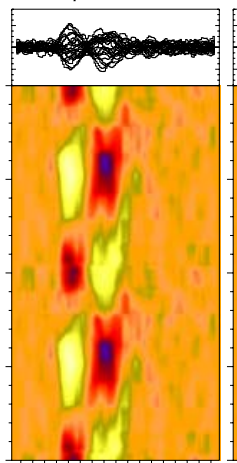

$\varphi=0.720$

$\varphi=0.823$
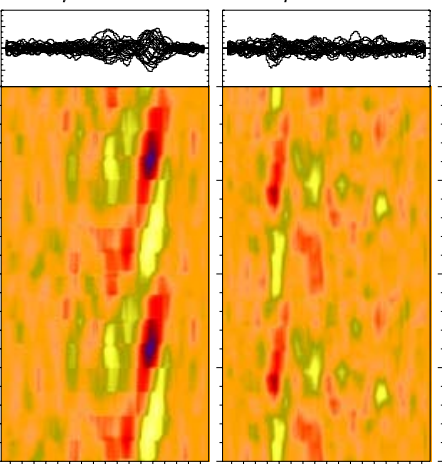

$\varphi=0.173$

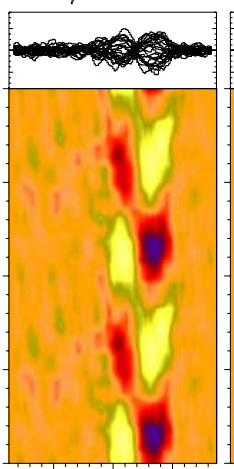

$\varphi=0.522$
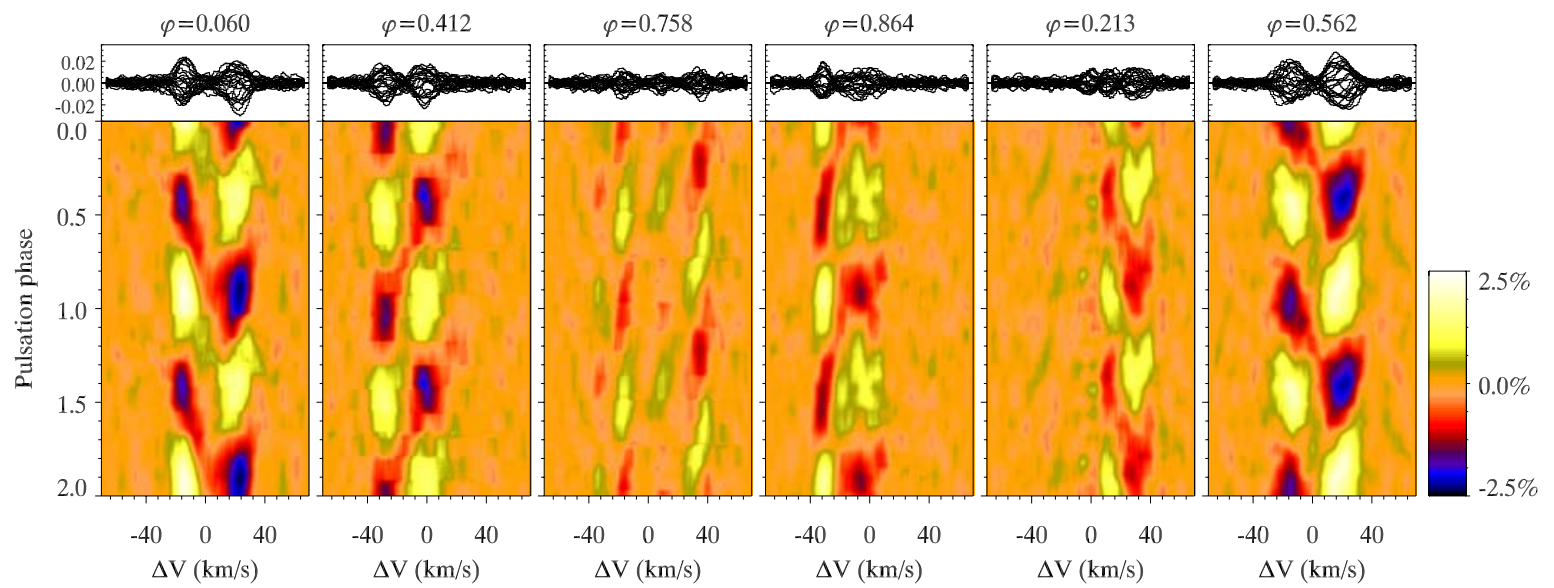

Fig. 3. Illustration of the Nd III $6145.07 \AA$ line profile variation at different rotation phases of HR 3831 . Each of the greyscale images is based on groups of 23 time-resolved spectra covering roughly 3 oscillation cycles of HR 3831 at specified rotation phases. The upper plot in each panel shows residuals from the mean line profile. The greyscale plots show time evolution of the residuals, phased with the main pulsation frequency $v=1427.9958 \mu \mathrm{Hz}$ and using the scale of $\pm 2.5 \%$ of the continuum intensity. The six columns of panels show LPV recorded during six different observing nights. The UT date is specified at the top of each column. Evolution of the variability pattern during observing nights is illustrated by three subpanels in each column which display spectra obtained at the beginning, middle, and end of the continuous monitoring.

corresponds to a moment when the area of relative $\mathrm{Nd}$ underabundance passes through the centre of the stellar disk. This explains the doubling of the average line profile and suggests that the complex oscillation behaviour at this phase is due to a transition from one pulsation pole to another. Due to moderately rapid rotation of the star and the respective substantial Doppler broadening, the areas associated with both pulsation poles are resolved in the wavelength domain and contribute to variability in the blue and red components of the $\mathrm{Nd} \mathrm{III}$ line. Despite low amplitude and the complex oscillation pattern observed at the crossover phases, pulsation does not vanish entirely and can still be detected easily with our high-precision observations.

An alternative representation of the $\mathrm{Nd}$ III line variability is shown in Fig. 3. These greyscale plots encompassing the whole dataset illustrate pulsational changes of the residuals with respect to the mean spectrum for the beginning, middle, and end of each of the six observing nights. The maximum deviation from the average spectrum reaches $\pm 2.5 \%$ of the stellar continuum. The pulsation pattern changes dramatically from one night to another. The data also show unambiguous evidence of systematic evolution of the variability pattern during individual 


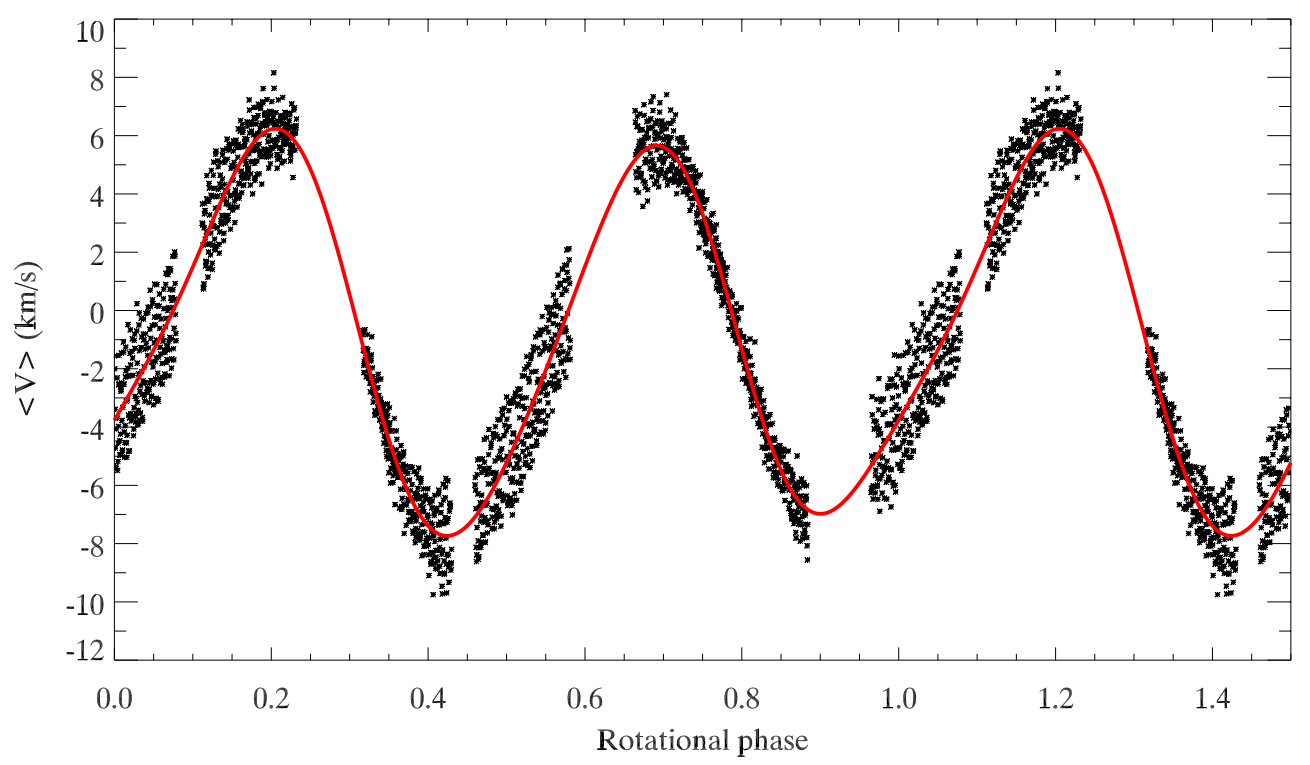

Fig. 4. Radial velocity variation of the Nd III $6145.07 \AA$ line in the spectrum of HR 3831. Symbols show individual measurements phased with the rotation period $P=2.851976$ d. The smooth curve illustrates a Fourier fit of the rotational modulation of the average RV.

observing nights. This captures rotational change in the aspect angle which becomes noticeable during a few hours of continuous monitoring (for instance, note an increase in variability on 6 Feb. 2001 and decrease on 14 Feb. 2001).

The spectrum variability plots presented in Figs. 2 and 3 establish a smooth pattern of the rapid line profile changes in HR 3831, similar to the pulsational variability observed in the atmospheres of many other types of stars pulsating with longer periods and low amplitudes. This observation represents a nontrivial result in light of the chemical stratification analysis of Ryabchikova et al. (2002). They demonstrate that the strong Nd III lines populating the roAp spectra form in tenuous high atmospheric layers, where physical conditions are rather uncertain and where some complex non-linear pulsation effects are possibly taking place. However, our observations do not provide any evidence of violent phenomena, such as supersonic shocks or large non-linearity associated with the propagation of pulsation waves. Consequently, we believe that, in the context of the present study, it is fully justified to carry out standard analysis of the pulsational spectroscopic observables by assuming that line profile changes are dominated by linear pulsation velocity fluctuations. At the same time, it should be acknowledged that the spectroscopic diagnostic explored in our paper is sensitive to the pulsational perturbations in the upper photosphere and the respective pulsation geometry is not necessarily the same as for the broad-band luminosity fluctuations originating in the lower part of the roAp atmospheres.

The data presented here is the first observation of the pulsational LPV in a rapidly rotating roAp star. The variability pattern found for the Nd III $6145.07 \AA$ line at the rotation phase of maximum pulsation amplitude is close to the one expected for an oblique dipolar oscillation (Kochukhov 2004a). The wave in the residual spectra propagates symmetrically from the blue to red line wing and then backwards. This behaviour differs from profile changes in the same Nd III line in the spectrum of the very slowly rotating roAp star $\gamma$ Equ. For this object Kochukhov \& Ryabchikova (2001a) discovered asymmetric variability characterised by the blue-to-red moving features only. However, observations of HR 3831 at the rotation phases of the magnetic crossover, or respectively minimum pulsation amplitude, reveal an LPV pattern which is more similar to the pulsational changes observed for $\gamma$ Equ. Variability is limited to a narrow wavelength region in one or both wings and the wave in the residual spectra propagates asymmetrically from blue to red (Fig. 3, 14 Feb.) or from red to blue (7 Feb.).

\subsection{RV measurements and frequency analysis}

A low angular degree of the non-radial mode excited in HR 3831 is reflected in a fairly simple pulsational line profile variation. Therefore, the integral observables, like the loworder line profile moments (Aerts et al. 1992), are sufficient for frequency analysis and should yield essentially the same information as pixel-by-pixel line profile measurements (e.g. Telting \& Schrijvers 1997). We determined moments of the Nd III $6145.07 \AA$ A line using expressions given by Kochukhov \& Ryabchikova (2001a), whose formulas are equivalent to the standard line profile moment definitions of Aerts et al. (1992).

The equivalent width $W_{\lambda}$, radial velocity $\langle V\rangle$, the second moment $\left\langle V^{2}\right\rangle$ (linked to the line width), and the third moment $\left\langle V^{3}\right\rangle$ (a measure of the line asymmetry) were all determined by integrating normalised spectra in the fixed $2 \AA$ window centred at the Nd III line. The relative precision of the RV measurements is considerably higher compared to $W_{\lambda}$ and other moments. Consequently, we chose $\langle V\rangle$ for detailed frequency analysis. The $1860 \mathrm{RV}$ determinations for the Nd III line are illustrated in Fig. 4, where the data are phased with the rotational ephemeris

$\mathrm{HJD}=2448312.23606+E \times 2.851976$

(Kurtz et al. 1997) that are used throughout our paper. An inhomogeneous $\mathrm{Nd}$ distribution causes a prominent double-wave 

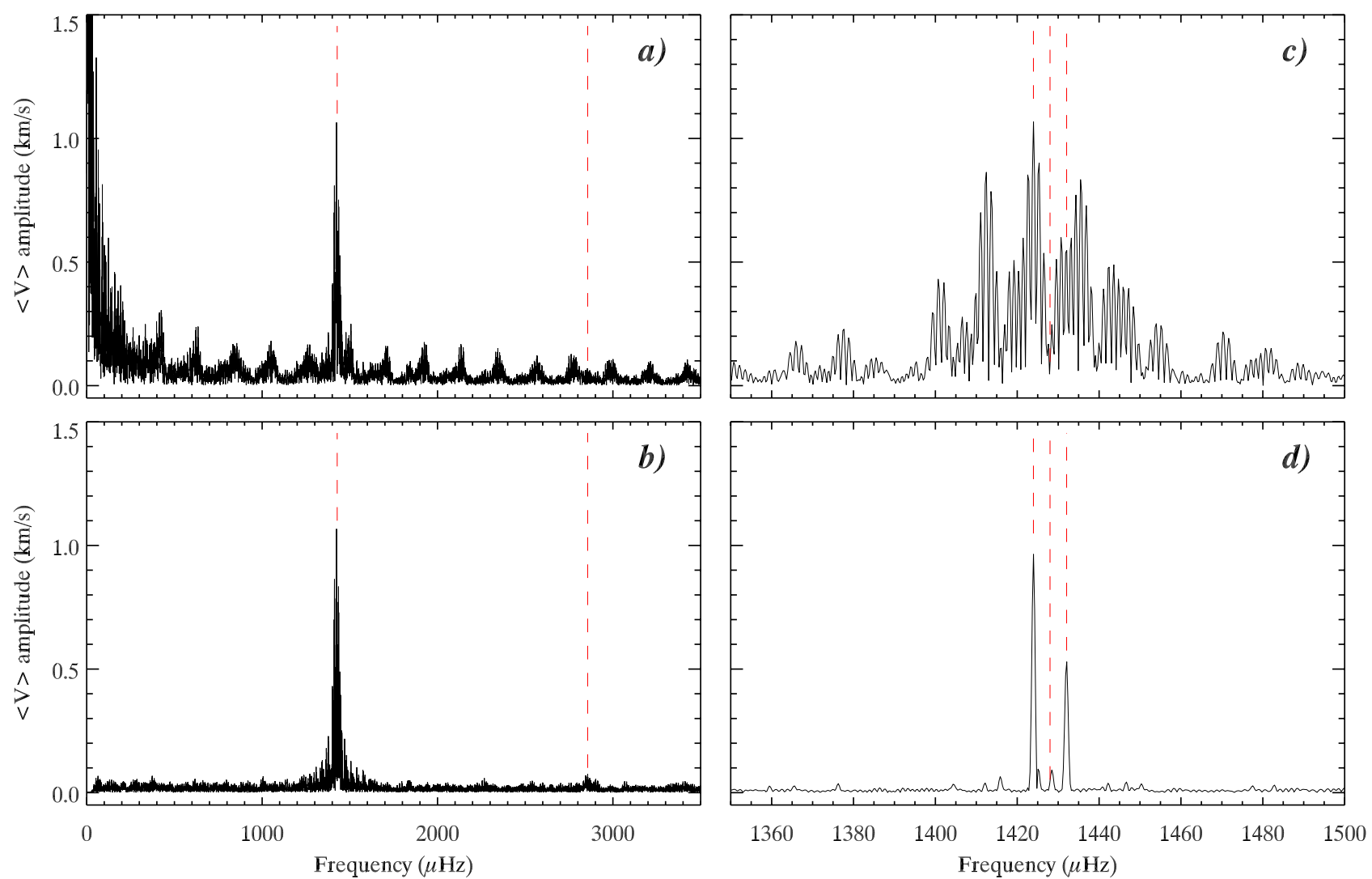

Fig. 5. Frequency analysis of the RV variation of the Nd III $6145.07 \AA$ line in the spectrum of HR 3831 . The left panels show lowresolution amplitude spectra. The vertical dashed lines mark the fundamental pulsation frequency $(v=1427.9958 \mu \mathrm{Hz})$ and its first harmonic $(2 v=2855.916 \mu \mathrm{Hz})$. a) The amplitude spectrum computed from the raw radial velocities shown in Fig. 4; b) the amplitude spectrum after prewhitening of the rotational modulation. The right panels present high-resolution amplitude spectra in the vicinity of the main pulsation frequency. The vertical dashed lines show the expected position of the triplet around the main frequency: $v-v_{\text {rot }}, v, v+v_{\text {rot }}($ where $v_{\text {rot }}=4058.265 \mathrm{nHz}$ ). c) The amplitude spectrum after prewhitening of the rotational modulation; d) the corresponding CLEANed amplitude spectrum.

rotational modulation of RV. Pulsational variability is superimposed on these longer term changes and manifests itself as a low-amplitude high-frequency scatter of the points in Fig. 4.

In the frequency domain (Fig. 5a), a contribution of nonradial pulsation is clearly seen as an excess of amplitude in the $1350-1500 \mu \mathrm{Hz}$ frequency range. In this paper we are primarily concerned with the pulsational changes of the spectrum of HR 3831, so we remove rotational modulation of RV by subtracting a 5-order Fourier fit (the smooth curve in Fig. 4) from the data in time domain. The amplitude spectrum after this prewhitening procedure is shown in Fig. 5b. The highamplitude peaks at low frequencies are gone and the pulsational contribution to the RV variability stands out with unprecedented clarity. A substantial pulsational signature in the $\langle V\rangle$ amplitude spectrum is only observed close to the main oscillation frequency of HR 3831. This differs from the photometric pulsational behaviour (Kurtz et al. 1997), where significant amplitude excess is also seen at the first and second harmonics.

The high-resolution amplitude spectrum (Fig. 5c) shows that aliasing remains a problem in our single-site data despite superb quality of the observational material. In a situation where the direct identification of the pulsation frequencies is hampered by the unfavourable window function, the
CLEAN technique (Roberts et al. 1987) often aids in distinguishing intrinsic pulsation frequencies from their aliases. We applied the CLEAN algorithm to the $\langle V\rangle$ measurements using 200 iterations with gain $=0.6$. The resulting amplitude spectrum (Fig. 5d) shows two high-amplitude peaks and a few weaker ones. Modification of the CLEANing parameters affects the low-amplitude peaks, but the two main frequencies remain unchanged and, thus, can be identified without any ambiguity. Their positions (1424.06 and $1432.03 \mu \mathrm{Hz})$ coincide with the $v-v_{\text {rot }}$ and $v+v_{\text {rot }}$ variation seen in the high-speed photometry. This observation provides a strong argument for the overall similarity between the frequency structure of the pulsational light and RV variation of HR 3831.

Given the satisfactory agreement between the main features of the spectroscopic and photometric pulsational variability at the fundamental frequency of HR 3831, we proceeded with the least-squares analysis by assuming that the oblique rotator model is valid for describing the $\langle V\rangle$ variation and that frequencies are always separated by multiples of $v_{\text {rot }}$ from the fundamental frequency $v$ and its harmonics. We used a nonlinear least-squares algorithm to fit the whole fundamental septuplet and the first-harmonic quintuplet. The respective 12 frequencies do not belong to physically different non-radial modes 
Table 2. Results of the non-linear least-squares fit of the Nd III $6145.07 \AA$ A line profile moment variations.

\begin{tabular}{|c|c|c|c|c|c|c|c|c|}
\hline \multirow[t]{2}{*}{ Frequency } & \multicolumn{2}{|c|}{$\overline{W_{\lambda}(\mu \AA)}$} & \multicolumn{2}{|c|}{$\langle V\rangle\left(\mathrm{km} \mathrm{s}^{-1}\right)$} & \multicolumn{2}{|c|}{$\left\langle V^{2}\right\rangle\left(\mathrm{km}^{2} \mathrm{~s}^{-2}\right)$} & \multicolumn{2}{|c|}{$\left\langle\left\langle V^{3}\right\rangle\left(\mathrm{km}^{3} \mathrm{~s}^{-3}\right)\right.$} \\
\hline & $A$ & $\varphi$ & $A$ & $\varphi$ & $A$ & $\varphi$ & $A$ & $\varphi$ \\
\hline \multicolumn{9}{|c|}{ The fundamental septuplet } \\
\hline$v-3 v_{\text {rot }}$ & $227 \pm 80$ & $0.242 \pm 0.056$ & $0.125 \pm 0.013$ & $0.180 \pm 0.018$ & $11.07 \pm 0.39$ & $0.392 \pm 0.006$ & $120 \pm 18$ & $0.557 \pm 0.024$ \\
\hline$v-2 v_{\mathrm{rot}}$ & $175 \pm 75$ & $0.625 \pm 0.069$ & $0.012 \pm 0.016$ & $0.806 \pm 0.166$ & $0.75 \pm 0.37$ & $0.665 \pm 0.079$ & $56 \pm 17$ & $0.554 \pm 0.049$ \\
\hline$v-v_{\mathrm{rot}}$ & $2643 \pm 74$ & $0.926 \pm 0.004$ & $1.079 \pm 0.013$ & $0.054 \pm 0.005$ & $8.30 \pm 0.36$ & $0.312 \pm 0.007$ & $877 \pm 17$ & $0.033 \pm 0.003$ \\
\hline$v$ & $249 \pm 79$ & $0.420 \pm 0.051$ & $0.025 \pm 0.015$ & $0.292 \pm 0.084$ & $0.73 \pm 0.39$ & $0.029 \pm 0.084$ & $56 \pm 18$ & $0.174 \pm 0.052$ \\
\hline$v+v_{\mathrm{rot}}$ & $1887 \pm 74$ & $0.851 \pm 0.006$ & $0.594 \pm 0.014$ & $0.983 \pm 0.006$ & $22.21 \pm 0.36$ & $0.723 \pm 0.003$ & $175 \pm 17$ & $0.967 \pm 0.016$ \\
\hline$v+2 v_{\mathrm{rot}}$ & $265 \pm 75$ & $0.232 \pm 0.045$ & $0.018 \pm 0.013$ & $0.550 \pm 0.165$ & $0.93 \pm 0.37$ & $0.293 \pm 0.063$ & $45 \pm 17$ & $0.805 \pm 0.061$ \\
\hline$v+3 v_{\text {rot }}$ & $34 \pm 80$ & $0.132 \pm 0.378$ & $0.051 \pm 0.013$ & $0.187 \pm 0.040$ & $3.81 \pm 0.39$ & $0.804 \pm 0.016$ & $141 \pm 18$ & $0.420 \pm 0.020$ \\
\hline \multicolumn{9}{|c|}{ The first-harmonic quintuplet } \\
\hline $2 v-2 v_{\text {rot }}$ & $118 \pm 74$ & $0.986 \pm 0.100$ & $0.038 \pm 0.012$ & $0.030 \pm 0.051$ & $1.34 \pm 0.36$ & $0.272 \pm 0.043$ & $3 \pm 17$ & $0.441 \pm 0.775$ \\
\hline $2 v-v_{\text {rot }}$ & $66 \pm 74$ & $0.808 \pm 0.178$ & $0.005 \pm 0.012$ & $0.920 \pm 0.386$ & $0.43 \pm 0.36$ & $0.552 \pm 0.131$ & $2 \pm 17$ & $0.661 \pm 1.211$ \\
\hline $2 v$ & $156 \pm 71$ & $0.950 \pm 0.072$ & $0.078 \pm 0.012$ & $0.996 \pm 0.026$ & $0.93 \pm 0.35$ & $0.391 \pm 0.060$ & $63 \pm 16$ & $0.956 \pm 0.042$ \\
\hline $2 v+v_{\text {rot }}$ & $108 \pm 74$ & $0.192 \pm 0.109$ & $0.006 \pm 0.012$ & $0.265 \pm 0.302$ & $0.52 \pm 0.36$ & $0.067 \pm 0.108$ & $4 \pm 17$ & $0.235 \pm 0.598$ \\
\hline $2 v+2 v_{\mathrm{rot}}$ & $193 \pm 74$ & $0.030 \pm 0.061$ & $0.044 \pm 0.012$ & $0.023 \pm 0.044$ & $1.50 \pm 0.36$ & $0.774 \pm 0.038$ & $35 \pm 17$ & $0.059 \pm 0.077$ \\
\hline
\end{tabular}

but arise from the rotational modulation of the same distorted oblique pulsation. The radial velocity is approximated with the expression

$$
\begin{aligned}
\langle V\rangle= & \sum_{i=-3}^{3} A_{i} \cos \left[2 \pi\left(\left(t-T_{0}\right)\left(v+i v_{\mathrm{rot}}\right)+\varphi_{i}\right)\right] \\
& +\sum_{j=-2}^{2} A_{j} \cos \left[2 \pi\left(\left(t-T_{0}\right)\left(2 v+j v_{\mathrm{rot}}\right)+\varphi_{j}\right)\right],
\end{aligned}
$$

where the epoch of phase zero is $T_{0}=2448312.23606$ and all amplitudes $A_{i, j}$, phases $\varphi_{i, j}$, and main pulsation frequency $v$ are optimised simultaneously. The search for the best-fit rotation frequency can also be carried out. The resulting value of the stellar rotation period, $P_{\text {rot }}=2.8624 \pm 0.0046 \mathrm{~d}$, agrees with $P_{\text {rot }}=2.851976 \pm 0.00003$ d determined by Kurtz et al. (1997), but is certainly less precise due to the shorter time-span of our observations. Hence, we preferred to adopt the constant photometric $v_{\text {rot }}=4058.265 \mathrm{nHz}$ in the fit with Eq. (2). The final parameters of the 12-frequency solution are reported in Table 2. Some of the pulsational contributions have amplitudes below the formal detection threshold but are, nevertheless, included here for completeness and to estimate of the upper limit of the RV variability at the respective frequencies. On the other hand, the second-harmonic triplet was not included in the fit because none of its components has an amplitude above $16 \mathrm{~m} \mathrm{~s}^{-1}$.

The fundamental pulsation frequency determined in the least-squares analysis is $v=1427.9958 \pm 0.0072 \mu \mathrm{Hz}$. This is the best determination of the main pulsation frequency of HR 3831. The comparison between observed RVs of the Nd III line and prediction of the frequency solution is illustrated in Fig. 6. Pulsational RV variation reaches a $3.6 \mathrm{~km} \mathrm{~s}^{-1}$ peak-to-peak amplitude, which is the highest value ever reported for a roAp star. For all observing nights, the stellar pulsational behaviour is modelled with good precision. The fundamental septuplet and the first-harmonic quintuplet together explain $86 \%$ of the data variance. The residual amplitude spectrum of $\langle V\rangle$ is shown in the upper panel of Fig. 7. The average noise level is $14 \mathrm{~m} \mathrm{~s}^{-1}$, whereas the highest high-frequency residual peaks reach $\approx 40 \mathrm{~m} \mathrm{~s}^{-1}$. In the low-frequency domain, we find a $59 \mathrm{~m} \mathrm{~s}^{-1}$ peak at $v=10.239 \mathrm{~d}^{-1}$ and its alias at $v=11.238 \mathrm{~d}^{-1}$. Detection of this variation is not statistically significant, but if not caused by the instrumental effects, it can be a candidate for a weak $\delta$ Scuti-type pulsation mode.

Although we found good overall concordance between the photometric and spectroscopic pulsation behaviour, the amplitudes and phases of the individual frequency components turn out to be quite dissimilar. The first striking discrepancy observed between the pulsational light variation and RVs is significantly more sinusoidal behaviour of the RVs. In fact, among the first-harmonic quintuplet components, only the $2 v$ variation is reliably detected, and its amplitude is 14 times lower than the amplitude of the largest component of the fundamental septuplet. Compare this with the time-resolved photometry in a Johnson $B$ filter (Kurtz et al. 1997), for which this ratio is 5 and even the second harmonic can be detected. Another difference is a substantially weaker main frequency component observed in the RV variation. A widely used diagnostic of the oblique pulsator geometry $\left(A_{+1}+A_{-1}\right) / A_{0}=\tan i \tan \delta$ (where $i$ is inclination angle the rotation axis and $\delta$ the mode obliquity relative to this axis, see Kurtz \& Martinez 2000) is 9.3, according to Kurtz et al. (1997). However, this parameter is 66.9 for our spectroscopic data, indicating that one or both characteristic angles are very close to $90^{\circ}$. The asymmetry between the amplitudes of the $v \pm v_{\text {rot }}$ pulsation components is also larger for RV: $A_{-1} / A_{+1}=1.82$ versus 1.21 in photometry.

\subsection{Moment variation and rotational modulation}

Analysis of the pulsational variation of the equivalent width and higher-order moments of the Nd III 6145.07 $\AA$ line uses an approach that is similar to the one applied to RV oscillations. The amplitudes and phases of the fundamental septuplet and first-harmonic quintuplet were optimised simultaneously. The main pulsation frequency was kept constant at the value of $\nu=1427.9958 \mu \mathrm{Hz}$ determined from the first moment. Results 

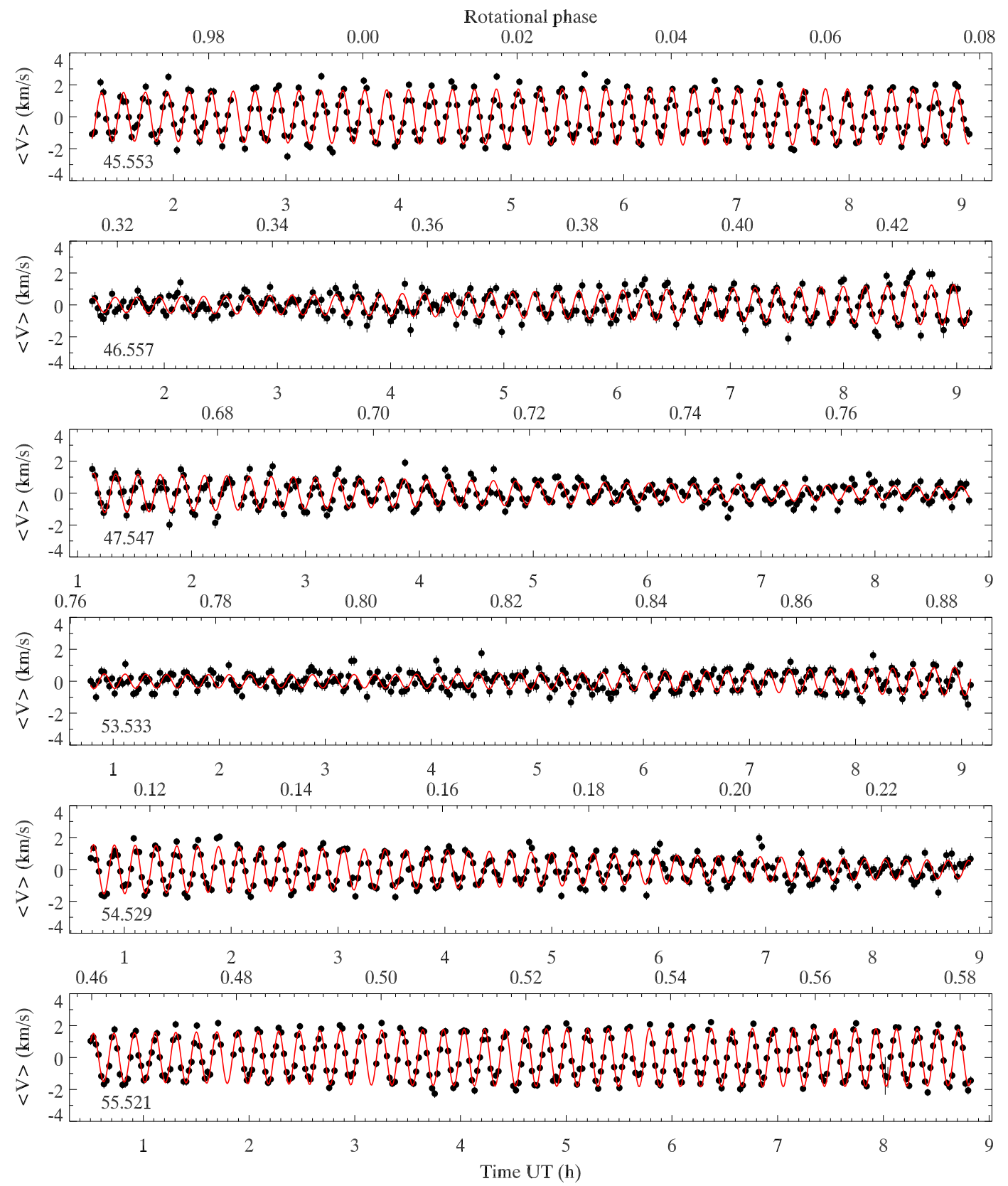

Fig. 6. Radial velocity curve for all observations of the Nd III $6145.07 \AA$ line in the spectrum of HR 3831. Symbols show individual RV measurements, with the rotational modulation of RV removed using the Fourier fit illustrated in Fig. 4. The solid curve shows the RV predicted by the frequency solution discussed in the text. Individual panels correspond to the data obtained during each of the six observing nights. The number in the lower left of each panel gives HJD -2 451900 at the start of spectroscopic monitoring. The $x$-axis is labelled with UT (in hours) and rotation phases.

of the fit of $W_{\lambda},\left\langle V^{2}\right\rangle$, and $\left\langle V^{3}\right\rangle$ are presented in Table 2 and illustrated in Fig. 7.

It is clear that the pulsational variation of all line profile observables considered here are detected with extremely high $S / N$ at the main pulsation frequency. Weak harmonic variability is also found at $\approx 4 \sigma$ level for the $\left\langle V^{2}\right\rangle$ and $\left\langle V^{3}\right\rangle$ moments. Inspection of the residual amplitude spectra of the line profile moments (Fig. 7) does not reveal any additional statistically significant amplitude peaks. Therefore, the frequency model defined by Eq. (2) is fully adequate and explains all variability present in the time series of the line profile moments.
An alternative presentation of the spectroscopic variability induced by the oblique non-radial pulsation in HR 3831 can be obtained by considering pulsation with only the main frequency $v$, but accounting for the rotational modulation by using a time-dependent amplitude and phase. This formulation corresponds closely to the actual pulsation behaviour thought to be present in HR 3831 and is mathematically equivalent to fitting constant amplitudes and phases of the fundamental septuplet components. Rotational modulation of the pulsations in $W_{\lambda}$, $\langle V\rangle,\left\langle V^{2}\right\rangle$, and $\left\langle V^{3}\right\rangle$ is illustrated in Fig. 8. The measurements shown in this figure were obtained by fitting the cosine function 

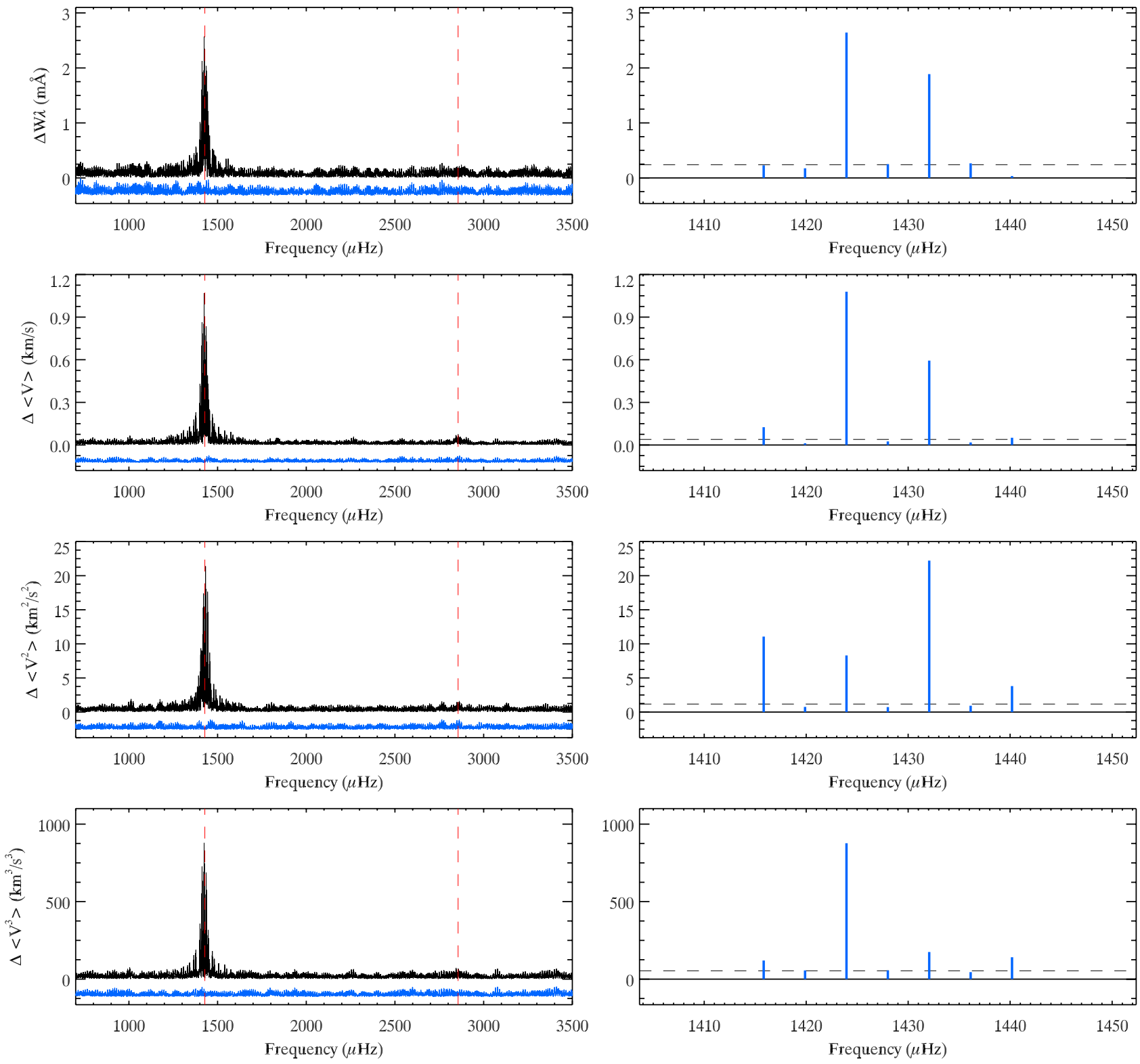

Fig. 7. Amplitude spectra of the pulsational variability of (from top to bottom) the equivalent width $W_{\lambda}$ and the first three moments - the radial velocity $\langle V\rangle$, the second moment $\left\langle V^{2}\right\rangle$, and the third moment $\left\langle V^{3}\right\rangle$ - measured for the Nd III 6145.07 $\AA$ line. The left panels show low-resolution amplitude spectra. The vertical dashed lines mark the main pulsation frequency and its first harmonic. The right sequence of panels illustrate amplitudes of the components of the fundamental septuplet $\left(v-3 v_{\text {rot }}, \ldots, v, \ldots, v+3 v_{\text {rot }}\right)$ derived with the least-squares analysis (see Table 2$)$. The horizontal dashed line corresponds to the $3 \sigma$ uncertainty of the fitted amplitudes. The lower amplitude spectra in the left panels show the amplitude spectra after the frequency solutions illustrated in the respective right panels have been removed from the data. These residual amplitude spectra are shown at the same scale as the original spectra and are shifted downward for display purposes.

to 47 subsets of our observations covering roughly 5 pulsation cycles. Rotational modulation of the line profile characteristics is well-defined and in perfect agreement with the modulation expected for the fundamental septuplet solution obtained above, confirming validity of both approaches to time-series analysis of the spectroscopic observations of HR 3831 .

The amplitude of the $W_{\lambda}$ variation reaches $\approx 5 \mathrm{~m} \AA$ at the rotation phase of the magnetic extrema. The phase modulation of the equivalent width changes is characterised by a sharper $\pi$ radian transition at the crossover phase compared to the $\langle V\rangle$ phase modulation. Thus, $W_{\lambda}$ shows more similarity to the photometric pulsational behaviour and, to some extent, can be used as a proxy for the latter.

The radial velocity amplitude modulation is qualitatively similar to the $W_{\lambda}$ amplitude changes, but does not show asymmetry between the two pulsation poles evident in the $W_{\lambda}$ data. We note that amplitude changes of the RV variation found in our paper are consistent with predictions by the generic oblique pulsator model. On the other hand, we do not confirm singlewave rotational modulation of the REE RV amplitude claimed by Balona (2002). Considering the large scatter of the measurements and a poor rotation phase coverage evident in Fig. 9 

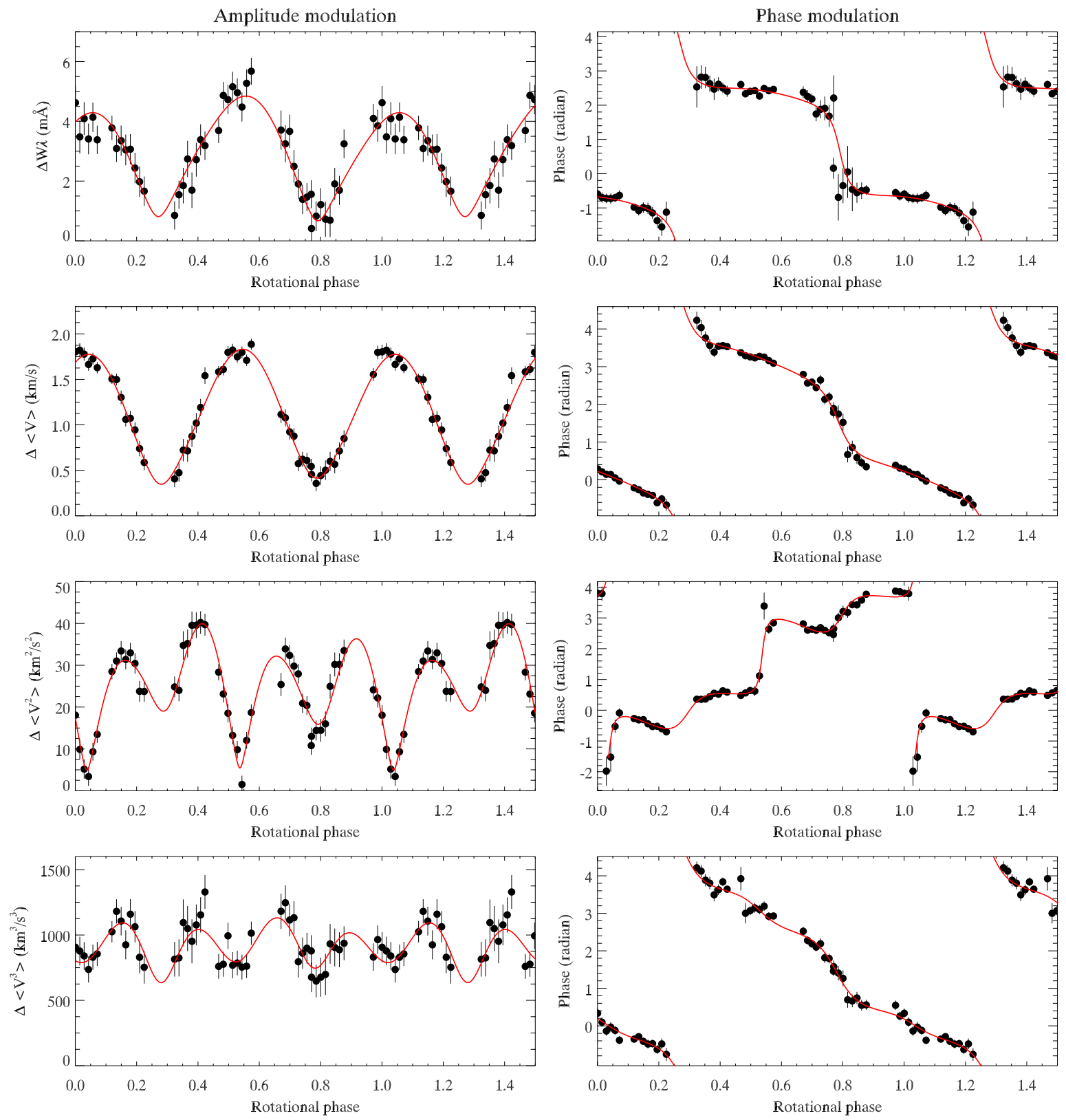

Fig. 8. Rotational modulation of the pulsation amplitude (left panels) and phase (right panels) of the variability in moments of the Nd III $6145.07 \AA$ line in the spectrum of HR 3831. The panels show (from top to bottom) measurements of the equivalent width $W_{\lambda}$, radial velocity $\langle V\rangle$, the second moment $\left\langle V^{2}\right\rangle$, and the third moment $\left\langle V^{3}\right\rangle$. Individual points in these diagrams were calculated by fitting the main pulsation frequency $(v=1427.9958 \mu \mathrm{Hz})$ to the subsets of 38 spectroscopic observations (about 5 pulsation cycles or 56 min). The smooth curves show rotational modulation expected from the frequency solution for the fundamental septuplet given in Table 2 and illustrated in Fig. 7.

of Balona's paper, we conclude that no rotational modulation of the RV oscillations was detected in his study and that the single-wave sinusoidal fit is not significant.

The second moment amplitude shows an unexpectedly complex rotational modulation with the two maxima and two minima during each rotation cycle. The minima observed at the phases of maximum RV are deeper and narrower compared to the decrease of $\left\langle V^{2}\right\rangle$ amplitude associated with the pulsational equator (rotation phases $\varphi \approx 0.25$ and 0.75 ). Below, we demonstrate that this interesting behaviour of the second moment is closely related to the distortion of oblique pulsation by the dipole component of the stellar magnetic field.
The amplitude maxima in the rotational modulation curves of $W_{\lambda}$ and $\langle V\rangle$ presented in Fig. 8 occur slightly later than predicted by the ephemeris of Kurtz et al. (1997). This $\Delta \varphi=$ $0.036 \pm 0.008$ discrepancy between the phases of the photometric and spectroscopic pulsation maxima may be intrinsic to the star or may indicate an error in the adopted rotation period. If the latter is true, $P_{\text {rot }}=2.852055 \pm 0.000017 \mathrm{~d}$ would make the time of the photometric pulsation maximum $(\mathrm{HJD}=2448312.23606$, Kurtz et al. 1997) agree with our spectroscopic observations. The improved rotation period deviates by less than $3 \sigma$ from the $P_{\text {rot }}$ derived by Kurtz et al. All results presented in our paper are based on the data covering 

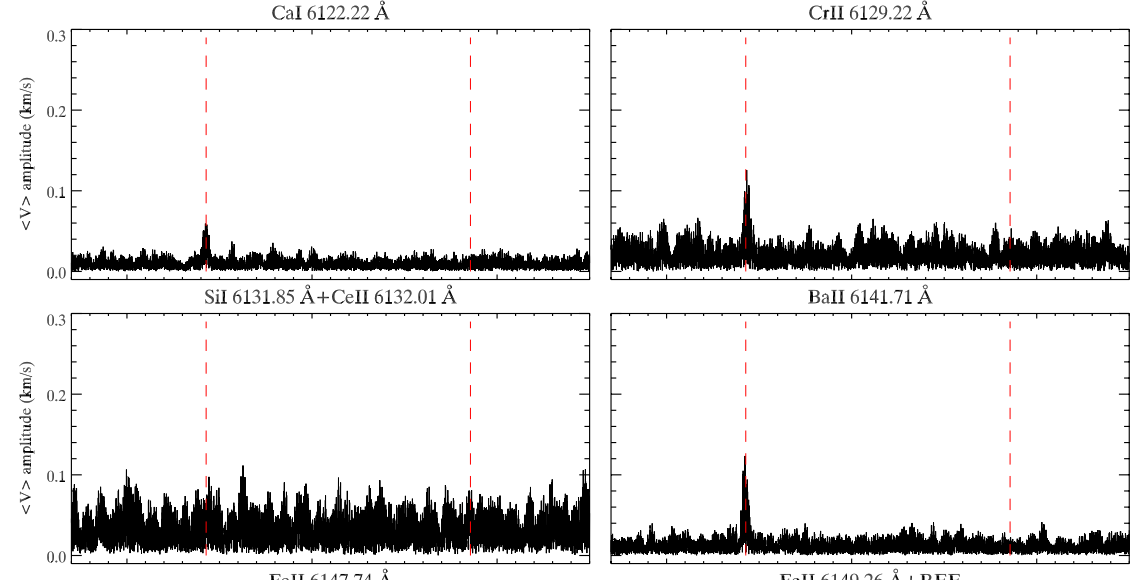

FeII $6147.74 \AA$
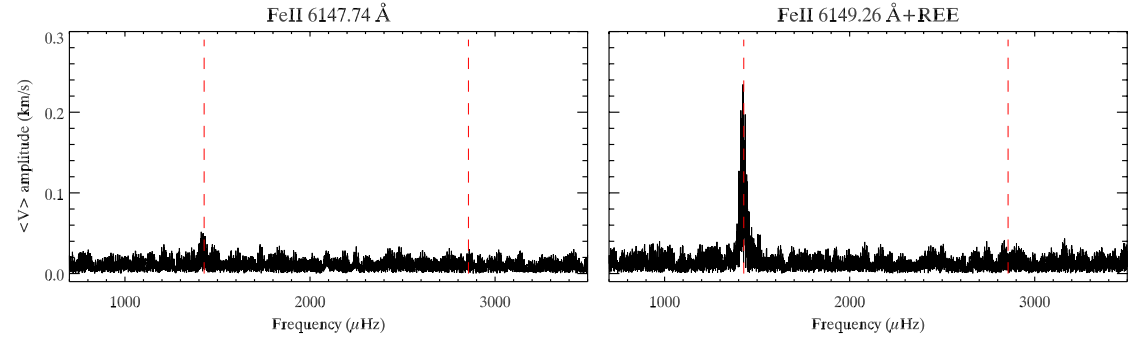

Fig. 9. Amplitude spectra of the RV variation observed for spectral features other than the Nd III $6145.07 \AA$ line. The vertical dashed lines mark the fundamental pulsation frequency $(v=$ $1427.9958 \mu \mathrm{Hz})$ and its first harmonic $(2 v=$ $2855.916 \mu \mathrm{Hz})$. just 3.6 stellar rotations and, therefore, are unaffected by this small correction to the rotation period.

\section{Pulsational changes in other lines}

Observations of HR 3831 analysed in this paper for the first time permitted measurements of pulsation amplitudes substantially below $50 \mathrm{~m} \mathrm{~s}^{-1}$ for individual spectral lines. Precision of our data enables detection of pulsational RV variation for essentially all strong and medium-strength lines. Analyses of these absorption features was carried out using the same procedure as was outlined above for the Nd III $6145.07 \AA$ line. Table 3 reports amplitudes of the components of the fundamental septuplet for 6 lines and blends in the 6120$6150 \AA$ spectral interval. Especially important is the first definite detection and measurement of the pulsational RV variation for the light and iron-peak elements, represented in the studied spectral region by the CaI $6122.22 \AA$ and Fe II $6147.74 \AA$ lines. The substantially larger amplitude of pulsations found for the Fe II $6149.26 \AA$ line reflects blending by an unidentified REE spectral line (cf. Kochukhov \& Ryabchikova 2001a). Oscillations are also easily detected for the Ba II $6141.71 \AA$ line, whose pulsation amplitude in HR 3831 is comparable to the pulsational changes of this line in the roAp star $\gamma$ Equ (Kochukhov \& Ryabchikova 2001a).

Our measurements of the RV variation for metal lines (Table 3) and the Nd III $6145.07 \AA$ spectral feature (Table 2) show a factor of 50 difference in amplitude and significant (sometimes as large as $\pi$ radian) discrepancy in phase. This picture is consistent with the general explanation of the pulsational spectroscopic behaviour of roAp stars proposed by Kochukhov \& Ryabchikova (2001a) and Ryabchikova et al. (2002): the running pulsation wave propagates outward through the chemically inhomogeneous stellar atmosphere with increasing amplitude. A large difference in the pulsation characteristics of various lines arises from interplay between the depthdependence of magnetoacoustic waves and chemical gradients formed by radiative diffusion. In particular, the rare-earth elements are concentrated in high atmospheric layers and probe regions with a large pulsation amplitude, whereas $\mathrm{Ca}$ and $\mathrm{Fe}$ lines originate from the deeper layers where pulsations are very weak. The strong Ba II lines are probably sensitive to the intermediate depths.

\section{Interpretation of moment variations}

The problem of modelling variations in the line profile moments for stars pulsating in oblique non-radial modes was addressed for the first time in a recent paper by Kochukhov (2005). In this study we derived the relevant expressions and developed a numerical procedure suitable for analysing the time-resolved spectroscopic observations of roAp stars. It was demonstrated that a non-axisymmetric superposition of the pulsation and rotation velocity fields in oblique pulsators leads to a qualitatively new behaviour for some of the line profile characteristics. Furthermore, a distortion of the oblique dipole modes predicted by the recent theoretical studies of the stellar magnetoacoustic oscillations (Bigot \& Dziembowski 2002; Saio \& Gautschy 2004) can be readily diagnosed through the moment analysis. In particular, the shape of the pulsation phase modulation for the radial velocity and second moment is very sensitive to non-axisymmetric pulsation components, whereas the rotational modulation of the second moment amplitude is best suited to revealing the axisymmetric, magnetically induced distortion of pulsations. The present paper deals with the first application of the new moment technique to observations of a real roAp star and aims at deriving a detailed model of the stellar pulsation velocity field.

The moment code of Kochukhov (2005) was employed to compute time-dependent amplitudes and phases of $\langle V\rangle$ and 
Table 3. Results of the non-linear least-squares fit of the RV variation of spectral lines other than Nd III $6145.07 \AA$.

\begin{tabular}{lccrcrc}
\hline \hline Frequency & \multicolumn{2}{c}{ Ca I $6122.22 \AA$} & \multicolumn{2}{c}{ Cr II $6129.22 \AA$} & \multicolumn{2}{c}{ Si I + Ce II $6131.9 \AA$} \\
& $A\left(\mathrm{~m} \mathrm{~s}^{-1}\right)$ & $\varphi$ & $A\left(\mathrm{~m} \mathrm{~s}^{-1}\right)$ & $\varphi$ & $A\left(\mathrm{~m} \mathrm{~s}^{-1}\right)$ & $\varphi$ \\
\hline$v-3 v_{\text {rot }}$ & $25 \pm 9$ & $0.511 \pm 0.055$ & $44 \pm 19$ & $0.509 \pm 0.068$ & $23 \pm 29$ & $0.484 \pm 0.206$ \\
$v-2 v_{\text {rot }}$ & $12 \pm 8$ & $0.446 \pm 0.108$ & $24 \pm 18$ & $0.289 \pm 0.116$ & $45 \pm 27$ & $0.036 \pm 0.098$ \\
$v-v_{\text {rot }}$ & $62 \pm 8$ & $0.439 \pm 0.021$ & $67 \pm 17$ & $0.211 \pm 0.041$ & $49 \pm 27$ & $0.470 \pm 0.087$ \\
$v$ & $27 \pm 9$ & $0.095 \pm 0.051$ & $21 \pm 18$ & $0.807 \pm 0.143$ & $35 \pm 29$ & $0.189 \pm 0.131$ \\
$v+v_{\text {rot }}$ & $53 \pm 8$ & $0.157 \pm 0.024$ & $115 \pm 17$ & $0.211 \pm 0.024$ & $81 \pm 27$ & $0.117 \pm 0.053$ \\
$v+2 v_{\text {rot }}$ & $10 \pm 8$ & $0.407 \pm 0.133$ & $28 \pm 18$ & $0.674 \pm 0.100$ & $38 \pm 27$ & $0.288 \pm 0.113$ \\
$v+3 v_{\text {rot }}$ & $13 \pm 9$ & $0.108 \pm 0.107$ & $43 \pm 19$ & $0.099 \pm 0.069$ & $51 \pm 29$ & $0.206 \pm 0.092$ \\
\hline Frequency & \multicolumn{2}{c}{ Ba II $6141.71 \AA$} & Fe II $6147.74 \AA$ & Fe II + REE $6149.26 \AA$ \\
\hline$v-3 v_{\text {rot }}$ & $41 \pm 11$ & $0.326 \pm 0.042$ & $41 \pm 10$ & $0.536 \pm 0.040$ & $86 \pm 12$ & $0.392 \pm 0.021$ \\
$v-2 v_{\text {rot }}$ & $14 \pm 10$ & $0.239 \pm 0.122$ & $15 \pm 10$ & $0.591 \pm 0.105$ & $47 \pm 11$ & $0.144 \pm 0.037$ \\
$v-v_{\text {rot }}$ & $132 \pm 10$ & $0.302 \pm 0.012$ & $45 \pm 9$ & $0.573 \pm 0.033$ & $248 \pm 11$ & $0.287 \pm 0.007$ \\
$v$ & $16 \pm 11$ & $0.018 \pm 0.109$ & $19 \pm 10$ & $0.895 \pm 0.085$ & $18 \pm 11$ & $0.779 \pm 0.103$ \\
$v+v_{\text {rot }}$ & $81 \pm 10$ & $0.173 \pm 0.020$ & $47 \pm 9$ & $0.093 \pm 0.032$ & $146 \pm 11$ & $0.985 \pm 0.012$ \\
$v+2 v_{\text {rot }}$ & $26 \pm 10$ & $0.567 \pm 0.064$ & $4 \pm 10$ & $0.530 \pm 0.398$ & $60 \pm 11$ & $0.563 \pm 0.029$ \\
$v+3 v_{\text {rot }}$ & $8 \pm 11$ & $0.269 \pm 0.210$ & $25 \pm 10$ & $0.205 \pm 0.064$ & $61 \pm 12$ & $0.007 \pm 0.030$ \\
\hline
\end{tabular}

$\left\langle V^{2}\right\rangle$ for 100 equidistant rotation phases. The local line profiles were approximated by a Gaussian with $\sigma=5 \mathrm{~km} \mathrm{~s}^{-1}$. An inclination angle $i=68.2^{\circ}$ and projected rotation velocity $v_{\mathrm{e}} \sin i=33.0 \mathrm{~km} \mathrm{~s}^{-1}$ were adopted following results of the model atmosphere and surface structure analysis of HR 3831 presented by Kochukhov et al. (2004). The free parameters of our spectroscopic oblique pulsator model included the obliquity $\delta$ and phase angle $\chi$ giving the orientation of the pulsation axis and amplitudes of the $\ell=1, m=0, \pm 1$ and $\ell=3$, $m=0$ pulsation components. These parameters were optimised through comparing the model predictions with the observed rotational modulation of the $\langle V\rangle$ and $\left\langle V^{2}\right\rangle$ amplitudes and the first moment phase. An equal weight was given to all three observables, and horizontal pulsation motions were neglected.

The final set of parameters of the oblique pulsator model required to fit variation of the Nd III $6145.07 \AA$ line profile moments is summarised in Table 4. Comparison between observations and model predictions is illustrated in Fig. 10. All considered pulsation observables, except the phase of $\left\langle V^{2}\right\rangle$, are either reproduced successfully ( $\langle V\rangle$ amplitude and phase) or fairly well $\left(\left\langle V^{2}\right\rangle\right.$ amplitude) by our model. It appears that complex modulation of the second moment amplitude is explained by the presence of the $\ell=3, m=0$ pulsation. It definitely cannot be reproduced by any combination of the dipole pulsation components (see Fig. 10) alone. We find that at the formation depth of the Nd III $6145.07 \AA$ line, the pulsation geometry is slightly non-axisymmetric, with the amplitude of the $\ell=1, m= \pm 1$ components not exceeding $\sim 15 \%$ of the contribution due to the $\ell=1, m=0$ spherical harmonic. On the other hand, we uncovered a prominent distortion of the dipole pulsation in HR 3831 by the global magnetic field. It manifests itself through the presence of a substantial axisymmetric octupole $(\ell=3, m=0)$ pulsation component and leads to enhancement of the velocity amplitude close to the pulsation poles. These findings agree well with the outcome of pulsation Doppler Imaging of HR 3831 (Kochukhov 2004b) and are in
Table 4. Parameters of the oblique pulsator model of HR 3831 determined from the variation of the Nd III $6145.07 \AA$ Aine profile moments. This table reports stellar inclination $i$, angles $\delta$ and $\chi$ specifying orientation of the pulsation axis, and amplitudes (renormalised to yield the maximum surface velocity) of different spherical harmonic contributions to the pulsation velocity field.

\begin{tabular}{cc}
\hline \hline Parameter & Value \\
\hline$i\left(^{\circ}\right)$ & 68.2 (fixed) \\
$\delta\left(^{\circ}\right)$ & $88.5 \pm 0.6$ \\
$\chi\left(^{\circ}\right)$ & $167.1 \pm 0.5$ \\
$A(\ell=1, m=0)\left(\mathrm{km} \mathrm{s}^{-1}\right)$ & $3.33 \pm 0.03$ \\
$A(\ell=1, m= \pm 1)\left(\mathrm{km} \mathrm{s}^{-1}\right)$ & $0.51 \pm 0.02$ \\
$A(\ell=3, m=0)\left(\mathrm{km} \mathrm{s}^{-1}\right)$ & $2.90 \pm 0.11$ \\
\hline
\end{tabular}

line with the theoretical predictions of Saio \& Gautschy (2004) and Saio (2005).

Precise information about the orientation of the pulsation axis obtained in our study demonstrates that oblique oscillations in HR 3831 are aligned with the dipolar magnetic field component. The latest and most detailed modelling of the magnetic topology of HR 3831 (Kochukhov et al. 2004) yielded magnetic obliquity $\beta=86.8 \pm 6.2^{\circ}$, which coincides with the pulsation mode obliquity $\delta=88.5 \pm 0.6^{\circ}$ determined through the moment analysis.

The present model describing variations of the Nd III $6145.07 \AA$ line cannot be improved by introducing high-order non-axisymmetric pulsation contributions. A formal least-squares analysis also yields the ratio of the horizontal to vertical pulsation amplitude, $K$, indistinguishable from zero. However, this result may reflect a failure of the standard constant $K$ parameterisation of the horizontal pulsation motions adopted in the moment technique. In fact, this approximation is poorly suited to characterise the latitude-dependent horizontal pulsation amplitude expected for magnetoacoustic pulsators (Saio \& Gautschy 2004). 

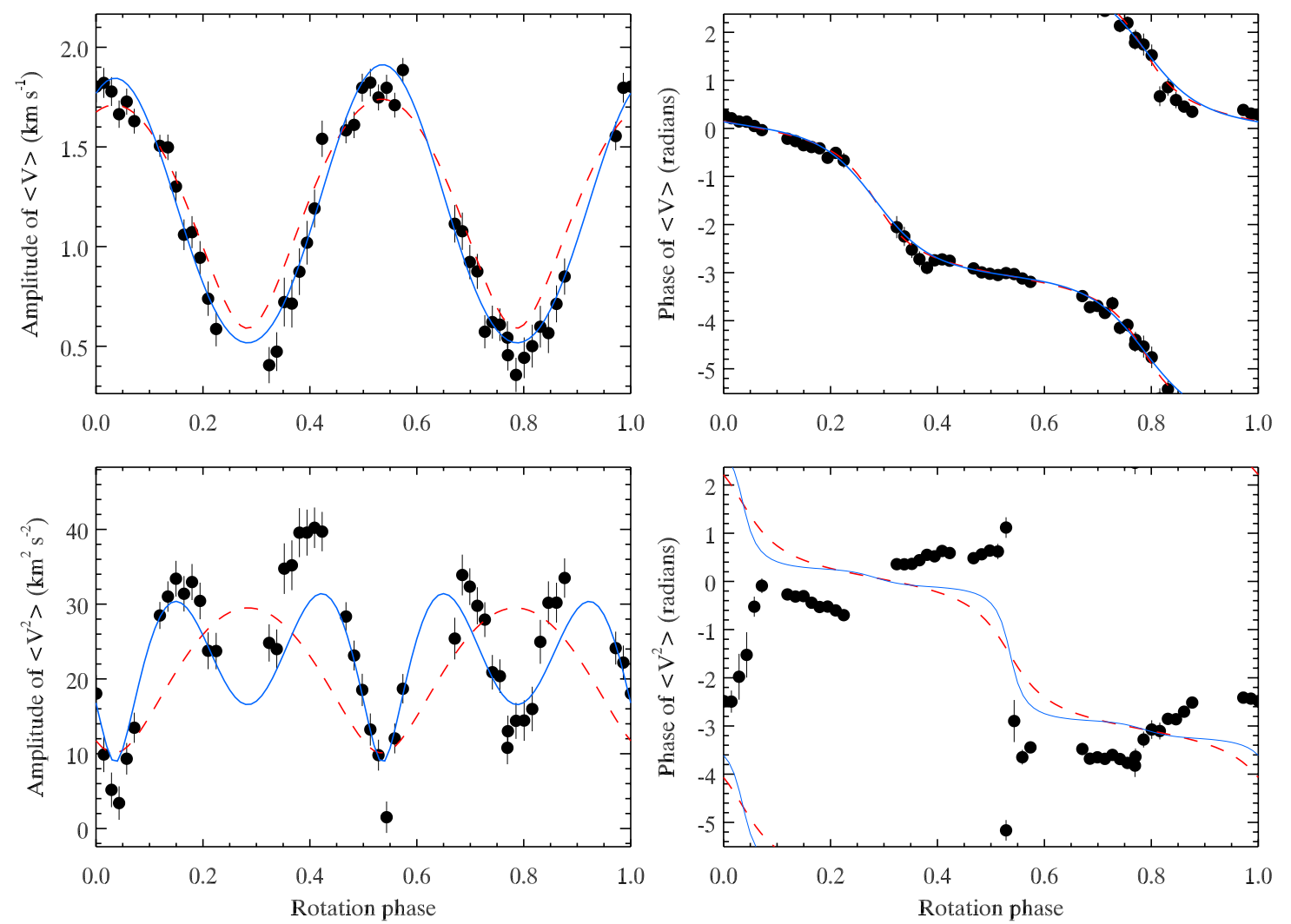

Fig. 10. Comparison of the observed rotational modulation of the pulsation amplitude and phase of the $\langle V\rangle$ and $\left\langle V^{2}\right\rangle$ moments of the Nd III $6145.07 \AA$ line (symbols) with predictions of the oblique pulsator model discussed in the text. The solid line shows results obtained with the best-fit combination of the non-axisymmetric dipole $(\ell=1, m=-1,0,1)$ and axisymmetric octupole $(\ell=3, m=0)$ pulsation components, whereas the dashed line illustrates the moment modulation expected from the dipole components alone.

The most significant limitation of the modelling described in this section is related to the fact that we disregard inhomogeneous distribution of $\mathrm{Nd}$ at the surface of HR 3831. The neodymium surface map reconstructed by Kochukhov et al. (2004) shows relative underabundance of this element along the magnetic equator. Consequently, the moment diagnostic is somewhat less sensitive to the pulsation wave properties in these regions of the stellar surface. However, we do not expect the results of the overall moment analysis to be significantly distorted by the presence of $\mathrm{Nd}$ spots, because in our modelling information is mostly extracted from the high-amplitude LPV observed at the rotation phases when no large Nd abundance gradients are present at the visible stellar disk.

\section{Discussion and conclusions}

Despite a long history of the high-speed photometric observations of pulsations in roAp stars, mechanisms responsible for the pulsational luminosity variation are still not understood. In particular, no theoretical model has been able to explain the observed complexity (non-linearity, peculiar wavelength dependence, etc.) of the photometric time series. Thus, no simple relation can be established between changes of luminosity and fundamental pulsation characteristics, such as the pulsation displacement vector. This explains why all interpretations of the photometric pulsational variability of roAp stars start with the assumption that a geometrical picture of the relative luminosity changes, $\delta I / I$, is the same as for the vertical component of the displacement vector and is given by a sum over spherical harmonic functions. Even the latest state-of-the-art theoretical investigations into pulsational light variability (e.g. Bigot \& Dziembowski 2002) have to abandon hopes of building a physically consistent picture and present an essentially geometrical model of the continuum intensity variation associated with $p$-mode oscillations. Progress in this field requires detailed non-adiabatic MHD modelling, with first such models being developed by Saio (2005), as a basis for estimating contributions of different terms to the observed luminosity variation of roAp stars. Nevertheless, the problem of low intrinsic diagnostic content for a disk-integrated photometric observable (cf. Saio \& Gautschy 2004) could never be overcome.

In contrast to unsatisfactory understanding and limited information content of the pulsational light curves, spectroscopic observations of rapid oscillations permit a straightforward astrophysical interpretation. The observed pulsation velocity fluctuation is directly related to the time dependence of the pulsation displacement vector. Given the absence of large pulsational changes in the strength of diagnostic lines, we can also assert that the observed RV variation is not visibly influenced by secondary effects, such as rapid changes in the temperature and density of atmospheric plasma. Consequently, interpretation of the spectroscopic observations of roAp 
pulsations offers us a real opportunity to develop physical models of magnetoacoustic pulsation for the first time since the discovery of roAp stars. Furthermore, the presence of strong vertical gradients of chemical composition in the atmospheres of roAp stars enables an unprecedented vertical resolution of pulsation waves. This makes spectroscopic studies of roAp pulsations a very promising research direction, both in terms of its enormous diagnostic potential and the possibility of establishing a physically realistic model of the pulsational phenomena from time-resolved observations.

Line profile analysis has become a standard tool for investigation into the pulsational variability of many types of oscillating stars. Yet due to their short pulsation periods, roAp stars have not been studied before with this technique. The present report on LPV in the prototype roAp star HR 3831 fills this gap and represents a significant step towards developing observational methods and analytical tools able to bring us closer to understanding the intricate nature of the magnetoacoustic pulsations in magnetic A stars.

Our line profile study of HR 3831 focuses on the variability of the Nd III $6145.07 \AA$ line, which shows maximum amplitude of the pulsational variability among spectral features in the wavelength region studied. It also exhibits one of the largest pulsation amplitudes ever observed in a roAp star. Spectroscopic material described in our paper shows unambiguous evidence of pulsational LPV and presents the very first comprehensive study of this phenomenon in a star pulsating in oblique non-radial mode. In particular, it enabled us to detect and characterise rotational modulation of the LPV pattern - an effect that was expected for roAp stars long ago (e.g. Baade \& Weiss 1987), but never observed until now.

Pulsational changes in the equivalent width and the first three moments of the $\mathrm{Nd}$ III line are detected with a very high $S / N$ ratio and studied over the whole stellar rotation cycle. Unlike strongly non-sinusoidal pulsation light curves (Kurtz et al. 1997), all spectroscopic observables show only a marginal presence of the first, and no signature of the second, harmonic in their amplitude spectra. Following Balona (2002), we interpret this observation as evidence that spectroscopy probes sinusoidal pulsations of the star, whereas variation of temperature and other parameters contributing to luminosity fluctuation are related to the pulsation displacement in a complex non-linear way.

Time-series analysis of the RV variation resulted in a remarkably accurate determination of the main pulsation frequency of HR 3831 and possible improvement of the rotation period of the star.

Taking advantage of the comprehensive observation of the LPV in the Nd III $6145.07 \AA$ line, we developed a physically consistent oblique pulsator model of HR 3831. This model directly characterises the horizontal geometry and amplitude of the pulsation displacement in the upper atmospheric layers where strong REE lines are formed. Rapid variation of the first two line profile moments is interpreted using a new version of the moment mode identification method (Kochukhov 2005). We derived the orientation of the pulsation axis and amplitudes of various spherical harmonic contributions to the pulsation geometry. These results generally agree with the pulsation Doppler Imaging map of the velocity field obtained for HR 3831 in our earlier study (Kochukhov 2004b).

Using the first detailed map of pulsations in a roAp star, we were able to test predictions of recent theoretical studies of magnetoacoustic pulsations. Two main types of distortion in the basic oblique dipole pulsator geometry were suggested. On one hand, Bigot \& Dziembowski (2002) take into account the interaction between weak magnetic field, pulsation, and rotation and predict a non-axisymmetric dipole pulsation geometry that is not aligned with the magnetic field axis. On the other hand, Saio \& Gautschy (2004) and Saio (2005) neglect stellar rotation and instead suggest that the dominant distortion of pulsation appears to be due to the strong magnetic field influence. Such a distortion manifests itself by the presence of substantial odd $\ell$ harmonic components in the pulsation geometry. Results of our moment analysis show a small (but significant) nonaxisymmetric contribution that could be ascribed to the $\ell=1$, $m= \pm 1$ components, but, contrary to suggestion by Bigot $\&$ Dziembowski (2002), we find no large misalignment of the pulsation and magnetic field axes in HR 3831. At the same time, we revealed a strong $\ell=3, m=0$ pulsation component, in good agreement with the model developed by Saio \& Gautschy (2004). This demonstrates that magnetic distortion of pulsation modes is by far a more important effect compared to the rotation and magnetic field coupling investigated by Bigot $\&$ Dziembowski (2002). Although a future comprehensive model of roAp pulsations should certainly include effects scrutinised by Bigot \& Dziembowski, neglect of the most important magnetic perturbation of $p$-modes in their modified oblique pulsator theory makes it inapplicable to roAp stars with average magnetic field strength above $\approx 1 \mathrm{kG}$, which includes HR 3831 . On the other hand, the success of the theoretical framework proposed by Saio \& Gautschy (2004) demonstrates that their model is based on a realistic description of the physics of magnetoacoustic pulsation and, hence, represents major progress in understanding excitation and propagation of pulsation waves in roAp stars.

The unprecedented precision of the time-series spectroscopic observations of HR 3831 analysed here permitted the first definite detection of weak pulsational RV changes in the lines of light and iron-peak elements. This makes it possible to attempt tomographic mapping (Kochukhov 2003) of the vertical structure of the pulsation mode in HR 3831. Such a procedure requires accurate information about the formation depths of the spectral lines involved in the analysis. Chemical stratification significantly affects the depths where lines of individual ions originate, which is why model atmosphere analysis and a stratification diagnostic are necessary prerequisites to reconstructing a realistic picture of the depth-dependence of pulsation characteristics. Such a study lies outside the scope of this paper and cannot be performed using spectra with the limited wavelength coverage available to us. Nevertheless, it is possible to probe certain general properties of the pulsation wave structure by using results of the tomographic mapping of pulsations in $\gamma$ Equ by Ryabchikova et al. (2002), which was mainly based on the same set of spectral lines as analysed here. The similarity of the atmospheric parameters and chemical composition of HR 3831 and $\gamma$ Equ suggests that the vertical abundance 

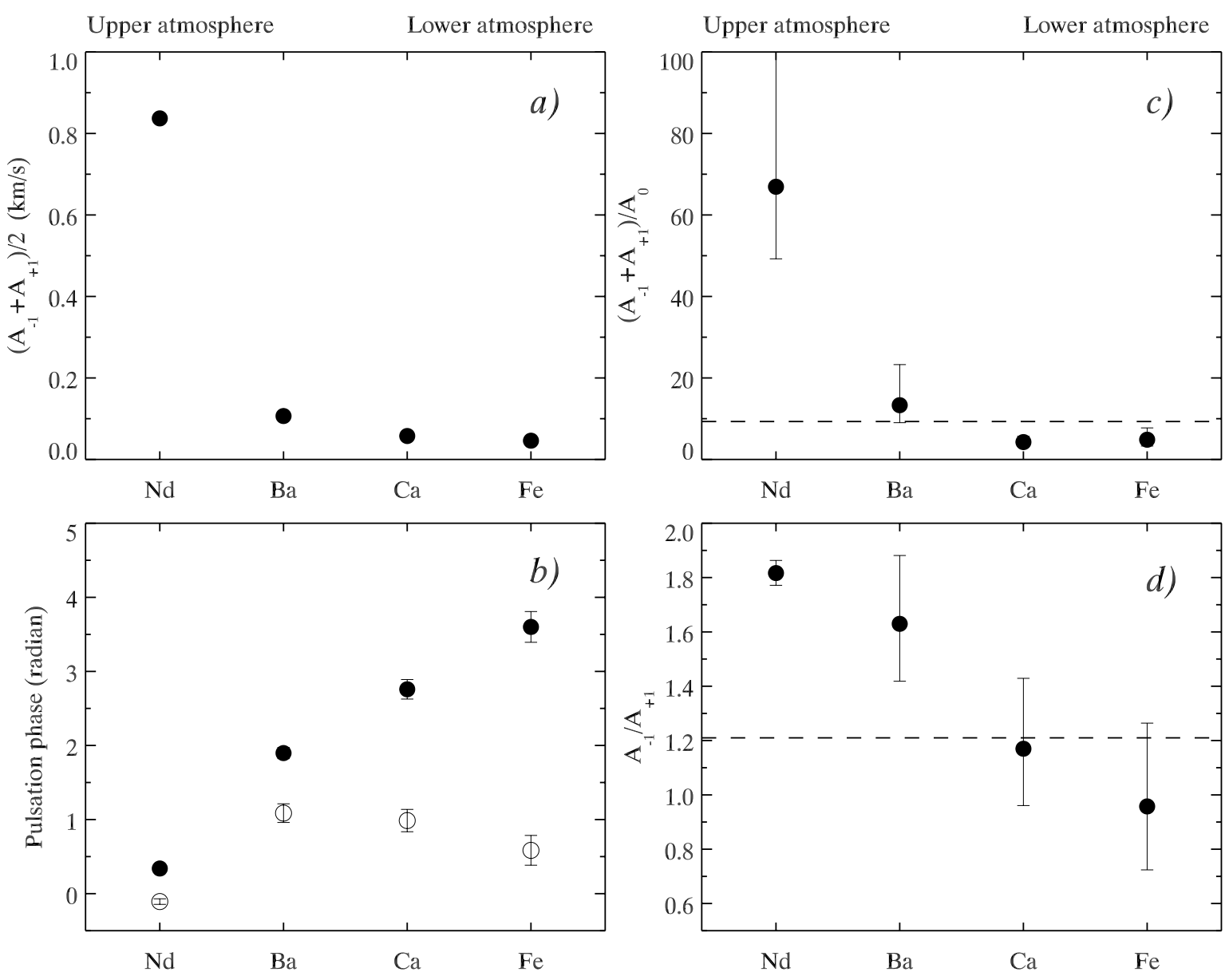

Fig. 11. Depth-dependence of the magnetoacoustic wave properties in the atmosphere of HR 3831 . a) $\left(A_{1}+A_{+1}\right) / 2$ characterises variation with depth of the average amplitude of the $v \pm v_{\text {rot }}$ components, b) depth-dependence of the respective phases of the $v+v_{\text {rot }}$ (open circles) and $v-v_{\text {rot }}$ (filled circles) pulsation, c) the $\left(A_{-1}+A_{+1}\right) / A_{0}$ parameter characterises strength of the main frequency component relative to the $v \pm v_{\text {rot }}$ components, d) $A_{-1} / A_{+1}$ measures asymmetry of the $v \pm v_{\text {rot }}$ components. The symbols show results obtained for individual spectral lines, whereas the dashed line corresponds to the photometric pulsation behaviour in a Johnson $B$ filter.

profiles and line-formation depths are comparable in the two stars. Thus, we can assert that the Nd III $6145.07 \AA$ line is formed in a high atmospheric layer and that the Ba II $6141.71 \AA$ line is sensitive to intermediate depths, whereas lines of $\mathrm{Ca}$ and Fe should originate in deeper layers. At the same time, the line of Ca I $6122.22 \AA$ investigated here is considerably stronger than Fe II 6147.71 $\AA$ and, hence, should form somewhat higher compared to the iron line. Using this tentative assignment of the relative formation depths of the four lines we were able to obtain the first picture of the vertical cross-section of the pulsation mode in HR 3831. Information about the depth-dependent behaviour of the amplitudes and phases of the fundamental triplet is summarised in Fig. 11.

Our observations establish that the amplitude of pulsations increases by a factor of 17 from the lower to upper layers in the atmosphere of HR 3831 (Fig 11a). Such behaviour is an expected consequence of the constancy (or at most small change) of the pulsation energy density $\rho v^{2} / 2$ in the presence of a rapid decrease in the gas density of a stellar atmosphere. The large pulsation phase difference between the RV variations of spectral lines sensitive to upper and lower atmospheric layers clearly points to a short vertical length for pulsation waves.
Comparison of the pulsation phases derived for different lines in the spectral region of HR 3831 studied here does not reveal a bimodal phase distribution but shows a quasi-continuous phase change with depth (Fig 11b). Therefore, we conclude that the vertical structure of pulsation in the atmosphere of HR 3831 resembles a running wave rather than a standing wave. With the data available here, we find no evidence of the existence of pulsation node in the atmosphere of HR 3831.

Comparison of our results with the theoretical nonadiabatic calculation (Saio 2005) shows reasonable agreement with respect to the magnitude of the pulsation amplitude increase towards the upper atmospheric layers and the absence of pulsation node in the line-forming region. However, a significant depth-dependence of the pulsation phase is not expected for this theoretical model.

In addition to probing depth-dependence of the average pulsation amplitude and phase, our measurements of the RV variation of the lines of different ions enable extraction of information about changes of the pulsation wave geometry with height. A common procedure for characterising oblique pulsator geometry and its distortion by the effects of stellar rotation is to study the asymmetry of the $v \pm v_{\text {rot }}$ peaks and estimate relative 
amplitude of the variation at the unshifted frequency $v$. The respective observables, $A_{-1} / A_{+1}$ and $\left(A_{-1}+A_{+1}\right) / A_{0}$, derived from the light curves of roAp stars have been interpreted in a number of theoretical investigations (see Kurtz \& Martinez 2000, for a recent overview) under the assumption that these parameters permit a simple geometrical interpretation within the oblique pulsator framework. Our study of HR 3831 reveals that, similar to the dramatic depth dependence of the pulsation amplitude and phase, relative amplitudes of the triplet components do not have well-defined values consistent with those inferred from the photometric observations of HR 3831, but they change significantly with height in the stellar atmosphere. In particular, analysis of the $\mathrm{Fe}$ II and $\mathrm{CaI}$ lines shows that pulsation in the lower part of the photosphere has moderate asymmetry for the $v \pm v_{\text {rot }}$ components and noticeable contribution of the pulsation at the main frequency $v$. Both characteristics agree with photometric results (Kurtz et al. 1997). However, the asymmetry of the rotationally separated peaks increases, and pulsation at the unshifted frequency decreases below the formal detection threshold when the diagnostic Nd III line sensitive to the upper atmospheric layers is considered (see Figs. 11c,d).

The pulsation behaviour observed for different lines in the HR 3831 spectrum can be attributed to a signature of the depth dependent superposition of the acoustic and magnetic pulsation wave components. Importance of the latter contribution presumably increases with height following the corresponding increase of the ratio of magnetic to gas energy density. Furthermore, in the lower part of the atmosphere, dissipation of the magnetic slow waves and related horizontal pulsation motions (Saio \& Gautschy 2004) could also contribute to the observed spectroscopic and photometric pulsation variability, thereby introducing further difference with oscillation as observed in the upper atmosphere using REE lines. Thus, a common notion that $A_{-1} / A_{+1}$ and $\left(A_{-1}+A_{+1}\right) / A_{0}$ are "geometrical" parameters typical of the whole pulsation structure is meaningless. Both quantities show significant variation with depth and should always be associated with specific layers of the atmosphere of a pulsating magnetic star. Thus, extreme caution is called for in a purely geometrical interpretation of the relative amplitudes of the $v, v \pm v_{\text {rot }}$ components.

Interpretation of the unique high time resolution spectroscopic data presented here inevitably calls for a significantly more complex 3-D picture of the structure of the pulsating cavity in roAp stars compared to the plane-parallel view provided by the traditional oblique pulsator paradigm. There is clearly a need to advance from a rudimentary phenomenological model to a self-consistent physical description of the propagation of magnetoacoustic waves in stellar atmospheres. The first important step in this direction is self-consistent modelling of the vertical and horizontal chemical inhomogeneities and pulsational variability using the pulsation Doppler Imaging method (Kochukhov 2004a,b). This modelling can be applied to spectral lines formed at different optical depths in order to deduce a 3-D structure of the pulsation velocity field. This modelling is currently underway for HR 3831 and will be presented in a forthcoming publication.
Acknowledgements. This study was partially supported by a postdoctoral stipend from the Swedish Research Fund and by the Lise Meitner fellowship from the Austrian Science Fund (FWF, project No. M757-N02).

\section{References}

Aerts, C., De Pauw, M., \& Waelkens, C. 1992, A\&A, 266, 294

Baade, D., \& Weiss, W. W. 1987, A\&AS, 67, 147

Baldry, I. K., Bedding, T. R., Viskum, M., Kjeldsen, H., \& Frandsen, S. 1998a, MNRAS, 292, 33

Baldry, I. K., Kurtz, D. W., \& Bedding, T. R. 1998b, MNRAS, 300, L39

Baldry, I. K., Viskum, M., Bedding, T. R., Kjeldsen, H., \& Frandsen, S. 1999, MNRAS, 302, 381

Baldry, I. K., \& Bedding, T. R. 2000, MNRAS, 318, 341

Balona, L. A. 2002, MNRAS, 337, 1059

Balona, L. A., \& Zima, W. 2002, MNRAS, 336, 873

Balona, L. A., \& Laney, C. D. 2003, MNRAS, 344, 242

Bigot, L., \& Dziembowski, W. A. 2002, A\&A, 391, 235

Cunha, M. S., \& Gough, D. 2000, MNRAS, 319, 1020

Cunha, M. S., Fernandes, J. M. M. B., \& Monteiro, M. J. P. F. G. 2003, MNRAS, 343, 831

Dziembowski, W. A., \& Goode, P. R. 1985, ApJ, 296, L27

Elkin, V. G., Riley, J. D., Cunha, M. S., Kurtz, D. W., \& Mathys, G. 2005, MNRAS, 358, 665

Houck, N. 1978, Michigan Spectral Catalogue, Vol. 2. University of Michigan, Ann Arbor

Hurly, P. R., \& Warner, B. 1983, MNRAS, 202, 761

Kanaan, A., \& Hatzes, A. P. 1998, ApJ, 503, 848

Kochukhov, O. 2003, in International Conf. Magnetic Fields in O, B and A Stars, ed. L. A. Balona, H. F. Henrichs, \& T. Medupe, ASP Conf. Ser., 305, 104

Kochukhov, O. 2004a, A\&A, 423, 613

Kochukhov, O. 2004b, ApJ, 615, L149

Kochukhov, O. 2005, A\&A, 438, 219

Kochukhov, O., \& Ryabchikova, T. 2001a, A\&A, 374, 615

Kochukhov, O., \& Ryabchikova, T. 2001b, A\&A, 377, L22

Kochukhov, O., Landstreet, J. D., Ryabchikova, T., Weiss, W. W., \& Kupka, F. 2002, MNRAS, 337, L1

Kochukhov, O., Drake, N. A., \& de la Reza, R. 2003, in IAU Symp. No. 210, Modelling of Stellar Atmospheres, ed. N. E. Piskunov, W. W. Weiss, \& D. F. Gray, ASP Conf. Ser., CD-D22

Kochukhov, O., Drake, N. A., Piskunov, N., \& de la Reza, R. 2004, A\&A, 424, 935

Kupka, F., Piskunov, N., Ryabchikova, T. A., Stempels, H. C., \& Weiss, W. W. 1999, A\&AS, 138, 119

Kurtz, D. W. 1978, Inf. Bull. Var. Stars, No. 1436

Kurtz, D. W. 1982, MNRAS, 200, 807

Kurtz, D. W. 1990, ARA\&A, 28, 607

Kurtz, D. W., Matthews, J. M., Martinez, P., et al. 1989, MNRAS, 240, 881

Kurtz, D. W., van Wyk, F., Roberts, G., et al. 1997, MNRAS, 287, 69

Kurtz, D. W., \& Martinez, P. 2000, Baltic Astron., 9, 253

Kurtz, D. W., Elkin, V. G., \& Mathys, G. 2003, MNRAS, 343, L5

Libbrecht, K. G. 1988, ApJ, 330, L51

Martinez, P., Weiss, W. W., Nelson, M. J., et al. 1996, MNRAS, 282, 243

Mashonkina, L., Ryabchikova, T., \& Ryabtsev, A. 2005, A\&A, 441, 309

Mathys, G. 1991, A\&AS, 89, 121

Mathys, G. 1995, A\&A, 293, 746 
Matthews, J. M., Wehlau, W. H., Walker, G. A. H., \& Yang, S. 1988, Ryabchikova, T. A. 2005, in IAU Symp., No. 224, The A-star ApJ, 324, 1099

Matthews, J. M., Kurtz, D. W., \& Martinez, P. 1999, ApJ, 511, 422

Medupe, R. 2002, Ph.D. Thesis, University of Cape Town Puzzle, ed. J. Zverko, J. Žižňovský, S. J. Adelman, \& W. W. Weiss, Cambridge University Press, IAUS, 224, 283

Mkrtichian, D. E., Hatzes, A. P., \& Kanaan, A. 2003, MNRAS, 345 781

Piskunov, N. E. 1999, in 2nd Workshop on Solar Polarization, ed. J. Stenflo, \& K. N. Nagendra (Dodrecht: Kluwer Academic Publishers), 515

Piskunov, N. E., \& Valenti, J. A. 2002, A\&A, 385, 1095

Saio, H., \& Gautschy, A. 2004, MNRAS, 350, 485

Savanov, I. S., Malanushenko, V. P., \& Ryabchikova, T. R. 1999, Astron. Lett., 25, 802

Schrijvers, C., Telting, J. H., \& Aerts, C. 2004, A\&A, 416, 1069

Shavrina, A. V., Polosukhina, N. S., Zverko, J., et al. 2001, A\&A, 372 571

Polosukhina, N. S., Shavrina, A. V., Hack, M., et al. 2000, A\&A, 357, 920

Roberts, D. H., Lehar, J., \& Dreher, J. W. 1987, AJ, 93, 968

Shibahashi, H., \& Takata, M. 1993, PASJ, 45, 617

Telting, J. H., \& Schrijvers, C. 1997, A\&A, 317, 723

Ryabchikova, T., Piskunov, N., Kochukhov, O., et al. 2002, A\&A, 384, Telting, J. H., Aerts, C., \& Mathias, P. 1997, A\&A, 322, 493 545

Thompson, I. B. 1983, MNRAS, 205, L43

Watson, R. D. 1988, Ap\&SS, 140, 255 